\title{
Effects of long-term microgravity exposure in space on circadian rhythms of heart rate variability
}

Yamamoto $\mathrm{N}^{1)}$, Otsuka $\mathrm{K}^{2)}$, Kubo $\mathrm{Y}^{1)}$, Hayashi $\mathrm{M}^{1)}$, Mizuno $\mathrm{K}^{3)}$, Tayama $\mathrm{I}^{4)}$, Ohshima $\mathrm{H}^{4)}$, Mukai $\mathrm{C}^{4)}$

1) Department of Medicine, Tokyo Women's Medical University, Medical Center East, Tokyo, Japan

2) Tokyo Women's Medical University, Department of Chronomics and Gerontology, Tokyo, Japan

3) Faculty of Child and Family Studies, Tohoku Fukushi University, Tokyo, Japan

4) Space Biomedical Research Office, Japan Aerospace Exploration Agency, Tokyo, Japan

\section{Abstract}

We evaluated their circadian rhythms using data from electrocardiographic records and examined the change in circadian period related to normal RR intervals for astronauts who completed a long-term ( $\geq 6$-month) mission in space. The examinees were seven astronauts, 5 men and 2 women, from 2009 to 2010. Their mean \pm SD age was 52.0 \pm 4.2 years (47-59 yr). Each stayed in space for more than 160 days; their average length of stay was 172.6 \pm 14.6 days (163-199 days). We conducted 24-hr Holter electrocardiography before launch (Pre), at one month after launch (DF1), at two months after launch (DF2), at two weeks before return (DF3), and at three months after landing (Post), comparing each index of frequency-domain analysis and 24-hr biological rhythms of the NN intervals (normal RR intervals). Results show that the mean period of NN intervals was within $24 \pm 4 \mathrm{hr}$ at each examination. Inter-individual variability differed among the stages, being significantly smaller at DF3 (Pre vs. DF1 vs. DF3 vs. Post=22.36 \pm 2.50 vs. $25.46 \pm 4.37$ vs. $22.46 \pm 1.75$ vs. $26.16 \pm 7.18 \mathrm{hr}, P<0.0001)$. The HF component increased in 2 of 7 astronauts, whereas it decreased in 3 of 7 astronauts and 1 was remained almost unchanged at DF1. During DF3, about 6 months after their stay in space, the HF component of 5 of 7 astronauts recovered from the decrease after launch, with prominent improvement to over $20 \%$ in 3 astronauts. 
Although autonomic nervous functions and circadian rhythms were disturbed until one month had passed in space, well-scheduled sleep and wake rhythms and meal times served as synchronizers. Key Words: Circadian period, Long space flight, Heart rate variability Short title: Change of circadian rhythms in long space flight 


\section{Introduction}

All life forms on Earth, from bacteria to human beings, have an internal body clock with circadian rhythms that are almost equal to the Earth’s rotation period (Dunlap, 1999). A living organism consisting of various levels of hierarchy monolithically structures the biological circadian rhythms to sustain its internal environment from external environmental disturbances (Ueda, 2005). In recent years, previous reports have described that a disordered body clock is linked with aging and a shortened lifespan as well as with diseases such as sleep disorders, carcinogenesis, obesity, and diabetes (Froy, 2011; Fu et al., Hurd and Ralph, 1998; 2002; Turek et al., 2005). Although these biological rhythms are reportedly affected by the external world, the life environment in space has only microgravity, which is vastly different from that on Earth. It also has no 24-hr lightdark cycle. Previous analyses of short-term space stays showed that a disordered circadian rhythm is associated with sleep disturbance and alterations in life rhythms (Gundel et al., 1997; Hirayanagi et al., 1997; Monk et al., 1998; Monk et al., 2001). Furthermore, many scientists expect that exposure to space environments that include microgravity might cause damage to important organs including the cardiovascular system (Eckberg et al., 2010; Gazenko et al., 1986). We only found one report describing changes in heart rate variability during a space mission of 6 months or longer. In that study, heart rate variability was reportedly improved through the long-term space stay (Verheyden et al., 2010). Our study examined 24-hr electrocardiographic records of astronauts on an extended mission in space, examining what effects the long-term stay had on their heart rate and autonomic nervous functions.

\section{Methods}

\section{Subjects}

The study subjects were 10 healthy astronauts (8 men, 2 women) who participated in ISS

Expeditions 19-25 from 2009 to 2010. Three of the ten subjects were excluded from these analyses 
because some of their data were missing. The mean ( \pm SD) age of the seven subjects was

$\mathbf{5 2 . 0 \pm 4 . 2 ~ ( 4 7 - 5 9 ) ~ y e a r s . ~ T h e i r ~ m e a n ~ s t a y ~ i n ~ s p a c e ~ w a s ~ 1 7 2 . 6 \pm 1 4 . 6 ~ d a y s ~ ( 1 6 3 - 1 9 9 ~ d a y s ) . ~ O n ~ t h e ~}$ average, astronauts had already experienced space flight $2.29 \pm 0.49$ times. The subjects were healthy adults who had passed NASA class III physical examinations. This study obtained all the subjects' consent and gained approval from the ethical committee jointly established by the Johnson Space Center and JAXA. A detailed explanation of the study protocol was given to the subjects before they gave written, informed consent, according to the Declaration of Helsinki Principles.

\section{RR interval data and heart rate variability}

To measure heart rate variability in space, we used portable 24-hr Holter electrocardiography monitors (FM-180; Fukuda Denshi). The device is small (65 [W] × 18 [D] × $62[\mathrm{H}] \mathrm{mm}, 78 \mathrm{~g})$ and useful in the tiny space of a spacecraft.

Measurements were made five times: once before a flight, three times during a flight (DF1, DF2, and DF3) and once after a flight. The before-flight measurement session (Pre) was conducted from 469 to 64 days before launch. The three measurement sessions during flight were taken on days $24 \pm 5$ (DF1) and 73 \pm 5 (DF2) after launch, and 15 \pm 5 days before return (DF3). The measurements after return were taken at 36 to 100 days (Post).

The measurement procedures and data collection were conducted as follows: Memory cards were inserted into the electrocardiograph. Five leads were placed on the precordium of the subjects and the electric power supply was connected to the main units of the electrocardiograph. The data stored in the memory card were downlinked to JAXA. Heart rate variability (HRV) analyses were conducted on Earth, with the RR intervals recorded by the 24-hr electrocardiograph. For HRV measurements, QRS waveforms were read from continuous electrocardiographic records. The RR intervals between normal QRS waveforms were extracted as the NN intervals. The measured NN 
intervals were A/D converted with $125 \mathrm{~Hz}$ (8 ms) time-resolution. The MemCalc/Chiram software (Suwa Trust GMS, Tokyo, Japan) was applied to these time series to remove artifacts. After the authors confirmed that all artifacts were actually removed and that the data excluded supraventricular or ventricular arrhythmia, time-domain and frequency-domain endpoints were computed and their circadian rhythms assessed (Hayano et al., 1990, Task Force of the European Society of Cardiology and the North American Society of Pacing and

Electrophysiology, 1996; Otsuka et al. 1997; Otsuka et al., 2000).

\section{Time-domain endpoints}

The Coefficient of Variation of R-R intervals (CVRR); root Mean Square Differences of successive R-R intervals (r-MSSD); Standard Deviation of the NN intervals (SDNN); Standard Deviation of the Averages of Normal Sinus to Normal Sinus (NN) intervals in all 5-minute segments of a 24-hr recording (SDANN (5 min)); and the Triangular Index (TI) were determined. The HRV of a short time segment of 5 min was used as an index reflecting autonomic nervous functions (Task Force of the European Society of Cardiology and the North American Society of Pacing and Electrophysiology, 1996). CVRR, r-MSSD, and SDANN were used for evaluation of every-5-min parasympathetic functions.

SDNN as an index of 24-hr long-time domain was used as the index related to life prognosis (lower values show worse life prognosis) (Bigger JT Jr et al., 1992; Task Force of European Society of Cardiology and the North American Society of Pacing and Electrophysiology, 1996; Penttilä J et al., 2001).

\section{Frequency-domain endpoints}

We assessed the high-frequency (HF), low-frequency (LF), and very-low-frequency (VLF) components over consecutive 5-minute segments over the entire 24-hr records. In other words, spectral analyses of 5-min time series of NN intervals served to determine the integrated spectral 
density over 3 frequency ranges corresponding to the HF (0.15-0.40 Hz), LF (0.04-0.15 Hz) and VLF (0.003-0.04 Hz) components. From the measured value of the LF and HF components, the ratio of LF to HF (LF/HF ratio) was calculated. The HF component was used for evaluation of sympathetic function. Sleep is deeply related to autonomic nervous functions. Parasympathetic nerves have important functions in REM sleep and parasympathetic nerves have important functions in non-REM sleep. Consequently, when the parasympathetic nervous functions during sleep are large, the time of non-REM sleep is sufficient, and the HF component is reported to be related to the quality of sleep (Busek et al. 2005; Hayano et al., 1996; Irwin et al., 2006). The input into a baroreceptor in blood vessels mainly consisting of the Mayor wave (sympathetic nervous activity) is adjusted by the baroreceptor reflex and is then output through the baroreflex-arc into sinus nodes as a fluctuation component, which is called the LF frequency component. The LF component consists of two components of parasympathetic and sympathetic activities. Therefore, the value obtained by dividing the LF component by the HF component (LF/HF ratio) was used as an index of activity balance between parasympathetic and sympathetic activities in the cardiovascular system.

VLF components are considered to represent endocrine activities, particularly the reninangiotensin system, which regulates vasomotor activities (Bernardi et al., 1996; Eckberg et al., 2005). Although they are reported to be indexes to reflect thermoregulation and although they are known to include fluctuation components of sleep apnea during sleep, this paper evaluated them as indexes representing endocrine activities. The ULF component is reportedly related to the inflammation reaction (CRP) and secretion of norepinephrine and cytokine (IL-6) (Aronson et al., 2001; Lampert et al., 2008). Because it is argued that ULF components should include nonperiodic (nonlinear) components, the background factors are too complex to discuss easily. Herein, we evaluated the ULF component as an index representing inflammation or immune activities. For 
ULF components, the indexes were measured by designating 180 min as one segment, since ULF components have much lower frequencies, in the range $<0.0033 \mathrm{~Hz}$ (periods of about 5-180 min).

\section{Circadian period analysis}

Targeting time series of 24-hr NN interval data, we assessed the presence or absence of circadian rhythms, using the MemCalc/Chiram (Suwa Trust GMS, Tokyo, Japan) HRV and biological rhythm analysis software (Sawada et al., 1997; Takusagawa et al., 1999). The MemCalc method starts analyses based on the existence of energy frequency distribution from the given finite time series data and then conducts spectral analyses using Burg’s Maximum Entropy Method (MEM), which includes the most spectral structures inherent in the data and which excludes any structure not contained in the time series. Thereafter, the multiple-component cosinor method was used to fit the MEM-based model to the data by least-squares. We determined whether one of several components detected in the MEM spectra had a period in the circadian range, defined as $24 \pm$ $4 \mathrm{hr}$ according to Halberg et al. (2009).

\section{Statistical analysis}

Data were expressed as mean \pm SD. Paired samples were analyzed by the Wilcoxon paired test, using the JMP (SAS Institute Inc., Cary, NC, USA). A p-value less than 0.05 was considered statistically significant.

\section{Results}

\section{Evaluation of autonomic activities and their transition during a long stay in space}

Figs. 1a-1d show results of one subject (ID01) who developed strong bradycardia at DF1. LF components decreased significantly during both sleep and waking spans compared with those before the flight $(\mathrm{P}<0.0001)$. The HF component, representing the quality of rest, decreased significantly (Fig. 1b). The VLF and ULF components increased significantly compared to pre- 
flight $(P<0.0001)$. VLF became large during sleep (Fig. 1b) and increased prominently for several hours immediately after wake-up ( $P=0.0001)$ (Fig. 1b).

Spectral analysis conducted after 6 months in space (DF3) shows that the LF component representing baroreceptor response was still lower than that recorded before the flight $(P<0.0001)$ (Fig. 1c). No significant difference was found as compared to DF1. The baroreceptor response started to decrease at DF1 and continued with no change during flight. By contrast, the HF component greatly improved compared with pre-flight and DF1 $(P<0.0001)$. In particular, the HF component during sleep increased markedly. Even in cases when it decreased at DF1, it improved during flight (Fig. 1c). VLF markedly increased as compared to pre-flight and continued unchanged $(P<0.0001)$ (Fig. 1c). Although no significant difference was found between the VLF components during sleep from that at DF1, it continued to increase significantly compared with that before flight. Immediately after wake-up, the ULF component increased further than during DF1 (P=0.0017) (Fig. 1c). Although after return the LF component became slightly lower than that before flight ( $P=0.049)$, it increased significantly compared with that during flight $(P<0.0001)$ (Fig. 1d), suggesting that it may have recovered after return. The HF component, representing the quality of rest, was mildly decreased compared with that before flight $(P=0.046)$, but it was much lower than that recorded during flight $(P<0.0001)$ (Fig. 3d). VLF recovered to the same level as that before flight. The prominent increase of the VLF component during sleep, observed during flight, became less pronounced (Fig. 1d). Although the ULF component still showed peaks several hours after wake-up as during the flight, this prominence decreased significantly after return $(P<0.0001)$ (Fig. 1d).

Figs. 1e-1h present results of another subject (ID04), who developed mild bradycardia at DF1. A significant decrease of the LF component was also found, similarly to the case depicted in Figs. 1ad (ID01) $(P<0.0001)$, but no significant change of the HF component was found. Unlike Figs. 1a-d, 
both ULF and VLF components became significantly lower as compared to pre-flight. In particular, ULF decreased greatly ( $P<0.0001)$. In late flight (DF3), although the ULF component remained significantly lower than that before flight, the LF, HF, and VLF components improved. In particular, the HF component was significantly increased compared with that before flight. Figs. 1i-1l show results of yet another subject (ID05), who developed mild tachycardia at DF1. The spectral analysis in early flight (DF1) revealed a decrease in the LF component $(P<0.0001)$. It was uniformly low during both sleep and waking (all night and all day); the HL component was also low all night and all day. Moreover, it increased markedly during the night. The VLF and ULF components decreased as compared to pre-flight $(P<0.0001)$. The ULF component was especially low at all times, showing no increase after waking, as had been seen before flight. During DF3, after 6 months in space, the heart rate improved to the level before flight. The circadian rhythm became more distinct compared with that during DF1. The LF and HF components did not recover and were lower than during DF1 $(P<0.0001)$. While showing tendencies to improve compared with DF1, the VLF and ULF components were still lower than before flight (Fig. 1-l).

\section{Change in indexes of heart rate variability of all astronauts}

Time-domain analyses of heart rate variability showed that r-MSSD and CVRR, representing parasympathetic activities, decreased in ID01 and improved in ID02 and ID03. In ID04 to ID06, parasympathetic activities decreased at DF1. Regarding the ratio of LF to HF, representing the balance between sympathetic activities and parasympathetic functions, sympathetic activities were dominant in ID01, ID03, ID05, and ID07, as were parasympathetic activities in ID02 and ID04. Furthermore, ID06 had no change. HF components are transmitted exclusively by parasympathetic nerves and used as index representing parasympathetic functions. In ID02 and ID03, they were increased at DF1, but decreased in ID01. In subjects showing mild bradycardia, they did not change 
at DF1 in ID04 and decreased to the level of 60\% of that before flight in ID05 (Table 1). In both individuals exhibiting tachycardia (ID05 and ID06), at DF1, the HF components decreased by 30\% as compared to pre-flight. On the other hand, during DF3, all individuals except for ID04 showed recovery of the HF component from the decrease at DF1. In addition, HF components in ID01, ID02, and ID03 increased by $20 \%$ or more as compared to pre-flight. Results after their return revealed no pattern because HF components varied among astronauts.

We observed the effect on, and the change in, SDNN, SDANN, and TI, presumed to represent life prognosis. Actually, SDNN at DF1 increased in five individuals: ID01, ID02, ID03, ID05, and ID07). However, SDNN was nearly unchanged in ID07 and decreased in ID04. During DF3, SDNN decreased below the level of those before flight in ID03 and ID06 but increased above that level in the other astronauts. Results show that SDNN (5 min) was 145.8 on average, changing from the lowest value of 72.3 to the highest value of 220.3. The SDANN (5 min) during flight was observed to increase by $20 \%$ or more in ID01, ID02 and ID03. Furthermore, they decreased in ID06 but increased mildly in ID07. In ID01, ID02 and ID03 it showed an increase by 20\% or more; SDANN after return also increased significantly in ID03 but decreased significantly in ID01 and ID02. In ID04 and ID05, with significant decrease of SDANN at DF3, SDANN improved after their return. In ID06, they improved in the late flight period but decreased again after return. In one individual (ID07), they were maintained with no change, but increased after return. SDANN (5 min) was 127.3 on average: the lowest value was 63.4 ; the highest value was 218.1 . At DF1, TI decreased in ID01 and ID04; increased in ID02, ID03, ID05 and ID07; and did not change in ID06. AtDF3, it decreased to near 70\% of that before flight in two individuals (ID01 and ID03) but did not decrease in one individual with high bradycardia (ID02). It decreased to 68\% in one individual (ID06) and changed only slightly compared with that before flight in another (ID07). Although the response of TI at DF1 varied, TI never decreased below 19, which is the level 
reported to be associated with a bad prognosis. The mean value was 34.8, with lowest and highest values of 21.3 and 58.0, respectively.

\section{Illustrative example of change in circadian rhythm of NN intervals}

Fig. 2 shows biological rhythms of RR intervals. It shows results of one subject (ID01) before flight (Figs. 2a, 2b), one month after lift-off (DF1) (Figs. 2c, 2d), after 6 months in space (DF3) (Figs. 2e, 2f), and after return (Figs. 2g, 2h). Spectral analysis and the least-squares fit of a multiple-cosine model based on major components detected by MEM were obtained for the NN interval data edited by mathematically removing noise from the RR interval series using the Mem/Calc software.

As Fig. 2a shows, before flight, the NN intervals average about $700 \mathrm{~ms}$ in the waking state, were gradually prolonged to 800-900 ms during sleep, and shortened again to $700 \mathrm{~ms}$ after waking up. Periods extracted from the MEM analysis include two large components with periods of $22.71 \mathrm{hr}$ and $11.80 \mathrm{hr}$, accounting for $31.6 \%$ and $34.9 \%$ of the variance, respectively (66.5\% total), Fig. $2 \mathrm{~b}$ (left). These two components are expected to represent the circadian rhythm. In addition, 7.31-hr and 5.30-hr components were also detected and used to approximate the circadian waveform accounting for $80.5 \%$ of the total variance (Fig. 2b, right).

At DF1 (Fig. 2c), the NN intervals vary around 700 ms during the waking state, which is not very different from that pre-flight. They lengthened to 900-1100 ms during sleep, which is longer than during pre-flight. Furthermore, NN intervals transiently dropped in association with exercise from 18:30 to 19:00. MEM analysis detected components with periods of $26.79 \mathrm{hr}$ (51.9\%) and $12.93 \mathrm{hr}$ (23.3\%), corresponding to the circadian and circasemidian components (Fig. 2d). These two large components are expected to represent the circadian rhythm characterizing the NN intervals. The record obtained after about 6 months in space (Fig. 2e) shows that NN intervals before sleep averaged about 700 ms, increased to 850-1000 ms during sleep (development of bradycardia), and 
decreased to 500-600 ms (development of tachycardia) at the time of awakening. The MEM analysis detects two major components with periods of $24.00 \mathrm{hr}$ and $11.68 \mathrm{hr}$ accounting for 35.6\% and 30.2\% of total power, respectively, with barely any other periodic component (Fig. 2f, left). The clear peaks at periods close to $24 \mathrm{hr}$ and $12 \mathrm{hr}$ suggest that the circadian clock mechanism had recovered from the disturbance immediately after flight, and had even improved compared with that before flight.

The reconstructed signal (Fig. 2f, right) shows a clear circadian variation. Analyses of NN intervals after return (Fig. 2g) show that they were 800-950 ms during sleep, less prolonged during sleep than in space, and recovered to the level before flight. In this case, the sleeping span was greatly shortened to about $3.5 \mathrm{hr}$. The MEM analysis detected components with periods of $18.17 \mathrm{hr}$ and $9.34 \mathrm{hr}$, accounting for $16.4 \%$ and $39.8 \%$ of total power, respectively, with barely any other periodic components (Fig. 2h, left). The short 9.34-hr component was the most prominent. Overall, the spectral power was markedly decreased. Whether the lack of a clear circadian rhythm is related to the short sleeping span remains to be determined.

\section{Circadian periodicity of all seven astronauts before, during and after flight}

Fig. 3 shows each astronaut's circadian period estimate of the NN interval data. On the average, the mean period varied within the circadian range of $24 \pm 4 \mathrm{hr}$ before, during, and after flight (Pre:

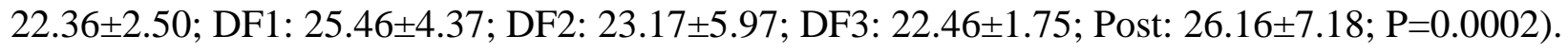

The inter-individual variation was significantly less during DF3.

\section{Discussion}

This observational study, conducted during a long-term space stay of at least 6 months, showed that although the circadian period became less marked after one month in space $(26.8 \pm 7.8 \mathrm{vs}$. 23.0 $\pm 1.7 \mathrm{hr}$ ), it improved, clustering more tightly around $24 \mathrm{hr}$ by the end of the stay. As reported earlier, exposure to a new environment such as space can greatly affect human physiology. In this 
study, after one month at the ISS, heart rate and autonomic activities, especially parasympathetic functions, changed greatly, as did NN intervals and the circadian period of autonomic nerves. The process by which the seven astronauts reacquired their circadian rhythms differed from one individual to another: three developed high bradycardia, two developed mild bradycardia, and two developed tachycardia in space as compared to pre-flight conditions. The difference in individual response patterns suggests that the capability of adapting to the space environment varies among individuals. Previous reports describe that a short-term stay in space is associated with a decrease in the baroreflex reflex arc, including baroreceptor and transient malnutrition (Eckberg et al., 2010; Hirayanagi et al., 2004). Responding to a decreased circulating blood volume, arteries tense and constrict to maintain blood volume. Sympathetic activities increase transiently, reducing parasympathetic activities (Gazenko et al., 1986). In this study, however, only four of seven individuals manifested increased sympathetic activities. In two other astronauts, parasympathetic activity increased. In other words, the response of the autonomic nervous system did not reflect the same tendency in all astronauts, but it did show that the method of response varied among individuals. In these two cases, it is considered that parasympathetic activities increased. In turn, sympathetic activities decreased so that HR decreased at DF1. However, in one of them (ID01) and in another case (ID04), it was the result of an abnormal accommodation of the low LF power. In the other case (ID05), both the parasympathetic and sympathetic activities were reduced. Mild bradycardia should hence be manifest as the sympathetic system changed more. In two cases (ID06, ID07), it is considered that parasympathetic activities were reduced to cause tension of the sympathetic nerve system and tachycardia, in keeping with previous reports (Gazenko et al., 1986). In this study, the ULF and VLF components highly increased in three individuals (ID01-03) during DF1. Astronauts come under strong stress immediately after launch into space. Therefore, the ACTH-cortisol system is activated at the level of nerves. Consequently, cortisol is increased 
(Leach et al., 1991). The highly activated ULF and VLF components in heart rate variability shown in our study suggest such responses in immune and endocrine systems. At last, there was a possibility that sampling rate of this study $(128 \mathrm{~Hz})$ affected the different result of $\mathrm{HRV}$ of each astronaut.

Moving to space, human beings become affected by the microgravity environment. According to previous reports, exposure to microgravity can lead to confused information input to the inner ear vestibule and muscle spindle or tendon (Schmitt H.H. et al., 1985), causing a central shift of fluids and thereby reducing heart and vascular regulatory functions.

In a microgravity environment, blood and fluid components move from extremities to the head, the great vessels in the chest, and the heart. As a result, blood pressure sensors in the carotid artery and the atrium work and feel an excessive increase of blood volume that must be regulated through the autonomic nervous and endocrine systems. The total blood volume is therefore reduced by $10 \%$ to 15\%. Blood components also change: even though erythrocytes decrease by almost 15\%, plasma volume is also decreased, so that they are effectively increased. Furthermore, erythropoietin secretion is decreased and the number of reduced erythrocytes is sustained. As renal circulation decreases, the RAA regulation system reportedly increases (Di Rienzo et al., 2008; Eckberg et al., 2012; Gazenko et al., 1986; Hirayanagi et al., 2004).

According to a study of circadian rhythm in intraoral temperature and agility of one cosmonaut who stayed at the Russian space station Mir, the influence of the endogenous circadian pacemaker on oral temperature and subjective alertness circadian rhythms was considerably weakened (Monk et al., 2001). A long-term bed down-tilt experiment conducted on volunteers showed that even if the lighting and feeding synchronizers were fully secured, circadian rhythms gradually deteriorated, resulting disorders became manifest, and the circadian acrophases of insulin and growth hormone were altered (S.L. Bonting., 1999). External changes such as gravity and light and dark cycles can thus affect important physiological functions (Pavy-Le Traon et 
al., 2007). In spite of such great external and internal changes, the seven astronauts who participated in this study all recovered a 24-hour-synchronized circadian rhythm after 6 months at the ISS.

Three factors may have helped the astronauts reacquire a 24-hour synchronized circadian rhythm.

First, regular light-dark and sleep-wake cycles are set when astronauts start living at the ISS, where lights are turned on at 7:30 GMT and turned off at 19:30 GMT. The illuminance during that time is 200-500 lux, which is effective for biological rhythms to adjust. It can be inferred that these conditions may be effective to recover biological rhythms. Second, since the feeding schedule can be expected to synchronize biological rhythms independently of the lighting schedule, establishing regular eating habits in space is important. Third, the increase of HF power during sleep may have played a role, as well-activated parasympathetic function during sleep improves sleep quality and increases the secretion volume of melatonin, which is likely to contribute to the improvement of circadian rhythms.

\section{Conclusion}

This study revealed that, although autonomic functions and the circadian period of NN intervals mediated by them were disordered after one month in space, regular sleep-wake and feeding schedules acting as synchronizers were successful in restoring proper circadian rhythms after 6 months at the ISS, despite the markedly different environment from that on Earth. The study further showed that processes leading to such adjustments differed among individual astronauts. 


\section{References}

Aronson D, Mittleman MA, Burger AJ. (2001). Interleukin-6 levels are inversely correlated with heart rate variability in patients with decompensated heart failure. J Cardiovasc Electrophysiol 12: 294-300.

Bernardi L, Valle F, Coco M, Calciati A, Sleight P. (1996). Physical activity influences heart rate variability and very-low-frequency components in Holter electrocardiograms. Cardiovasc Res 32: 234-237.

Bigger JT Jr, Fleiss JL, Rolnitzky LM, Steinman RC. (1992). Time course of recovery of heart period variability in patients with myocardial infarction and ventricular arrhythmias. Am J Cardiol 69: 718-723.

Busek P, Vanková J, Opavský J, Salinger J, Nevsímalová S. (2005). Spectral analysis of the heart rate variability in sleep. Physiol Res 54: 369-376.

Di Rienzo M, Castiglioni P, Iellamo F, Volterrani M, Pagani M, Mancia G, Karemaker JM, Parati G. (2008). Dynamic adaptation of cardiac baroreflex sensitivity to prolonged exposure to microgravity: data from a 16-day spaceflight. J Appl Physiol 105: 1569-1575.

Dunlap JC. (1999). Molecular bases for circadian clocks. Cell 96: 271-290.

Eckberg D, Kuusella T. (2005). Human vagal baroreflex sensitivity fluctuates widely and rhythmically at very low frequencies. J Physiol 567:1011-1019.

Eckberg DL, Halliwill JR, Beightol LA, Brown TE, Taylor JA, Goble R. (2010). Human vagal baroreflex mechanisms in space. J Physiol 588: 1129-1138.

Froy O. (2011). Circadian rhythms, aging, and life span in mammals. Physiology 26: 225-235.

Fu L, Pelicano H, Liu J, Huang P, Lee CC. (2002). The circadian gene Period 2 plays an important role in tumor suppression and DNA damage response in vivo. Cell 111: 41-50. 
Gazenko OG, Shulzhenko EB, Egorov AD. (1986). Cardiovascular changes in prolonged space flights. Acta Physiol Pol 37: 53-68.

Gundel A, Polyakov VV, Zulley J. (1997). The alteration of human sleep and circadian rhythms during spaceflight. J Sleep Res 6: 1-8.

Halberg F, Cornélissen G, Wilson D, Singh RB, De Meester F, Watanabe Y, Otsuka K, Khalilov E. (2009). Chronobiology and chronomics: detecting and applying the cycles of nature. Biologist (London) 56:209-214.

Hayano J, Sakakibara Y, Yamada M, Kamiya T, Fujinami T, Yokoyama K, Watanabe Y, Takata K. (1990). Diurnal variations in vagal and sympathetic cardiac control. Am J Physiol 258: H642-646. Hayano J, Yasuma F, Okada A, Mukai S, Fujinami T. (1996). Respiratory sinus arrhythmia. Phenomenon improving pulmonary gas exchange and circulatory efficiency. Circulation 94: 842847.

Hirayanagi K, Iwase S, Kamiya A, Sasaki T, Mano T, Yajima K. (2004). Functional changes in autonomic nervous system and baroreceptor reflex induced by 14 days of 6 degrees head-down bed rest. Eur J Appl Physiol 92: 160-167.

Hurd MW, Ralph MR. (1998). The significance of circadian organization for longevity in the golden hamster. J Biological Rhythm 13: 430-436.

Irwin MR, Valladares EM, Motivala S, Thayer JF, Ehlers CL. (2006). Association between nocturnal vagal tone and sleep depth, sleep quality, and fatigue in alcohol dependence. Psychosom Med 68: 159-166.

Lampert R, Bremner JD, Su S, Miller A, Lee F, Cheema F, Goldberg J, Vaccarino V. (2008). Decreased heart rate variability is associated with higher levels of inflammation in middle-aged men. Am Heart J 156: e1-7. 
Leach CS, Cintron NM, Krauchs JM. (1991). Metabolic changes observed in astronauts. J Clin

Pharmacol 31: 921-927.

Monk TH, Buysee DJ, Billy BD, Kennedy KS, Willrich LM. (1998). Sleep and circadian rhythm in four orbiting astronauts. J Biol Rhythms 13: 188-201.

Monk TH, Kennedy KS, Rose LR, Linenger JM. (2001). Decreased human circadian pacemaker influence after 100 days in space: a case study. Psychosom Med 63: 881-885.

Otsuka K, Ichimaru Y, Cornélissen G et al. (2000). Dynamic analysis of heart rate variability from 7-day Holter recordings associated with geomagnetic activity in subarctic area. Computers in Cardiology 27: 453-456.

Otsuka K, Nishimura Y, Kubo Y et al. (1997). Chronomes (rhythms, chaos and age trends) of human heart rare variability in both genders. Computers in Cardiology 24: 49-52.

Pavy-Le Traon A, Heer M, Narici MV, Rittweger J, Vernikos J. (2007). From space to Earth: advances in human physiology from 20 years of bed rest studies (1986-2006). Eur J Appl Physiol 101: 143-194.

Penttilä J, Helminen A, Jartti T, Kuusela T, Huikuri HV, Tulppo MP, Coffeng R, Scheinin H. (2001). Time domain, geometrical and frequency domain analysis of cardiac vagal outflow: effects of various respiratory patterns. Clin Physiol 21: 365-376.

Sawada Y, Ohtomo N, Tanaka Y, Tanaka G, Yamakoshi K, Terachi S, Shimamoto K, Nakagawa M, Satoh S, Kuroda S, Iimura O. (1997). New technique for time series analysis combining the maximum entropy method and non-linear least squares method: its value in heart rate variability analysis. Med Biol Eng Compt 33: 2821-2831.

Schmitt HH, Reid DJ (1985). Anecdotal information on space adaptation syndrome. NASA/Space Biomedical Research Institute and University Space Research Association Division of Space Biomedicine, July, 1-21. 
S.L. Bonting (1999). Advances in Space Biology and Medicine 7

Takusagawa M, Komori S, Umetani K, Ishihara T, Sawanobori T, Kohno I, Sano S, Yin D, Ijiri H, Tamura K. (1999). Alternations of autonomic nerve activity in recurrence of variant angina. Heart 82: 75-81.

Task Force of the European Society of Cardiology and the North American Society of Pacing and Electrophysiology. Heart rate variability: standards of measurement, physiological interpretation, and clinical use. (1996). Circulation 93: 1043-1065.

Turek FW, Joshu C, Kohsaka A, Lin E, Ivanova G, McDearmon E, Laposky A, Losee-Olson S, Easton A, Jensen DR, Eckel RH, Takahashi JS, Bass J. (2005). Obesity and metabolic syndrome in circadian Clock mutant mice. Science 308: 1043-1045.

Ueda HR, Hayashi S, Chen W, Sano M, Machida M, Shigeyoshi Y, Iino M, Hashimoto S. (2005). System-level identification of transcriptional circuits underlying mammalian circadian clocks. Nature Genetics 37: 187-192.

Verheyden B, Liu J, Beckers F, Aubert AE. (2010). Operational point of neural cardiovascular regulation in humans up to 6 months in space. J Appl Physiol 108: 646-654. 
Fig. 1-a

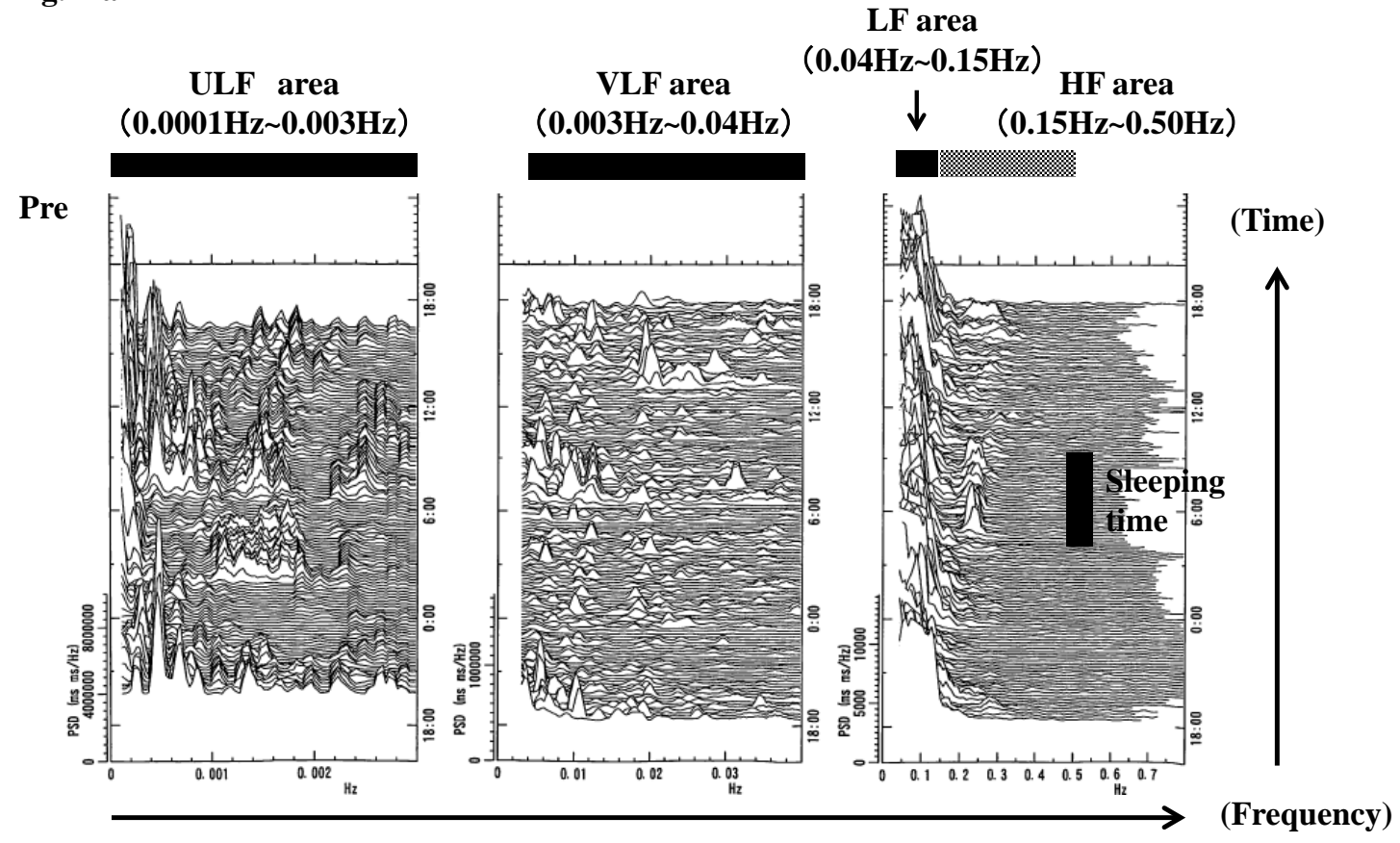

Fig. 1-b

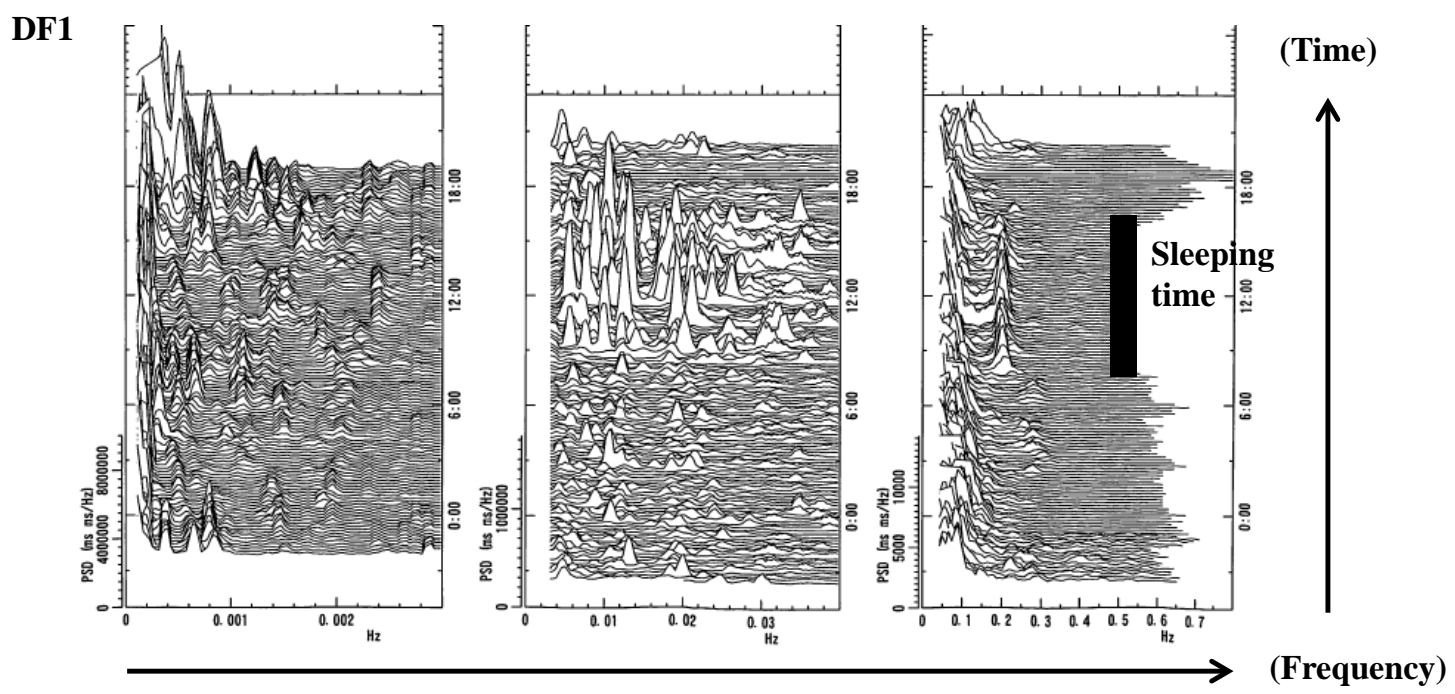


Fig. 1-c

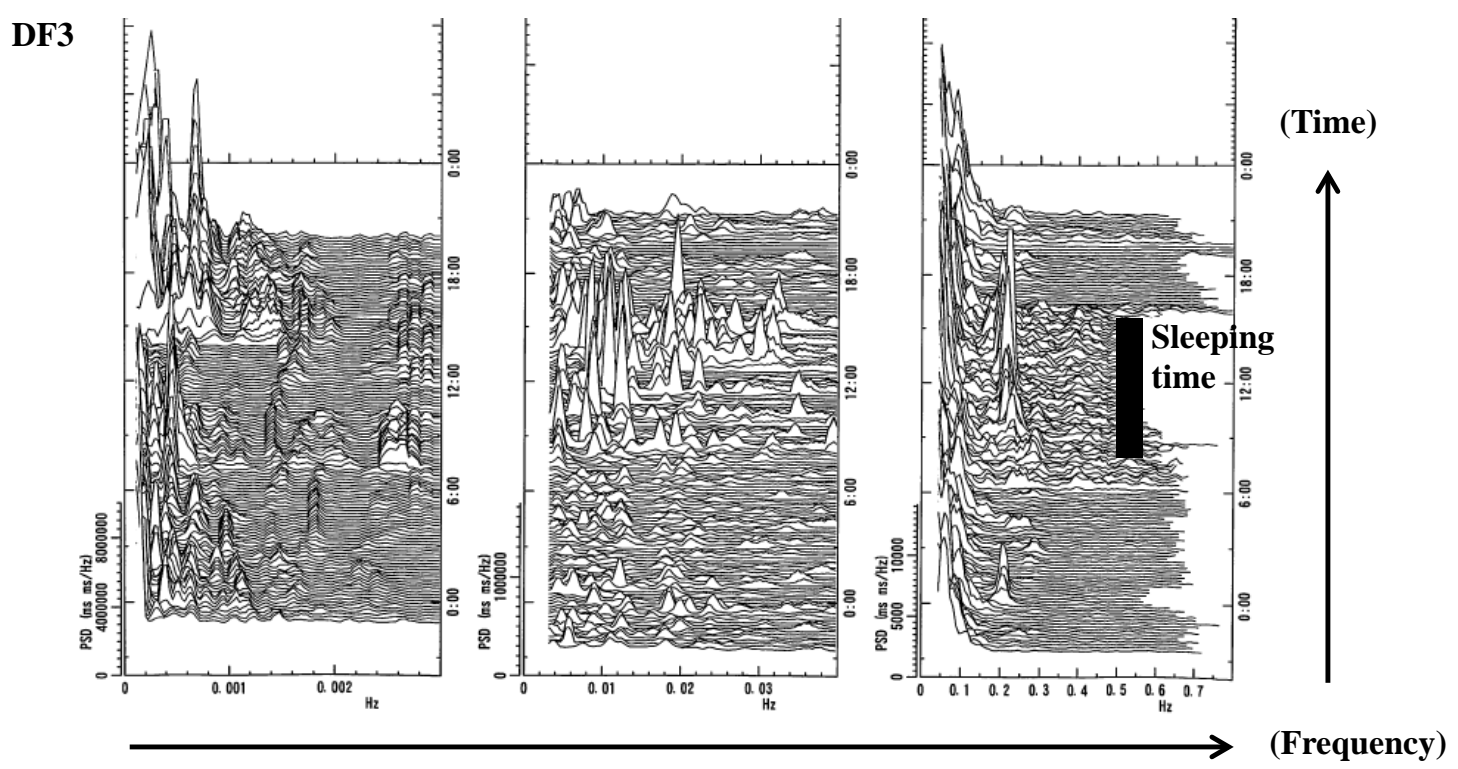

Fig. 1-d

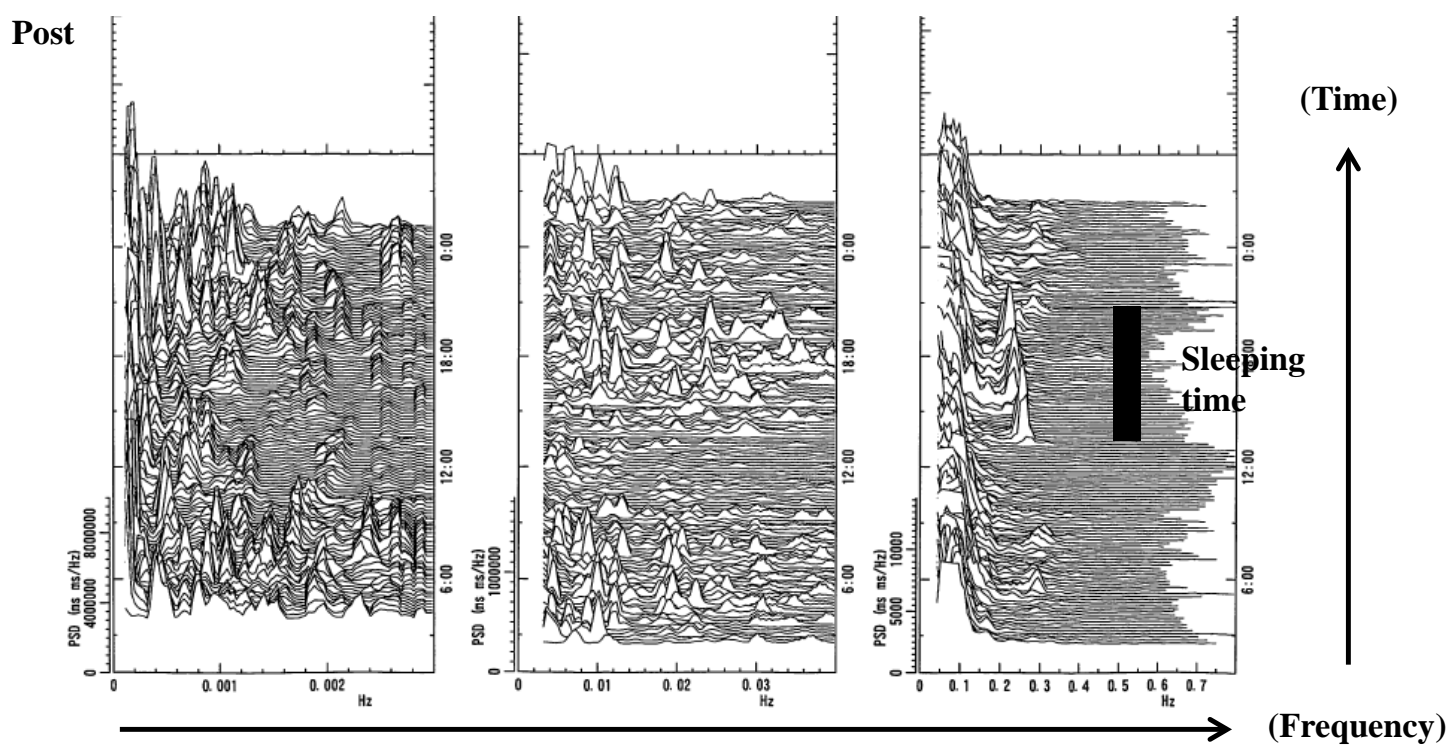


1-e
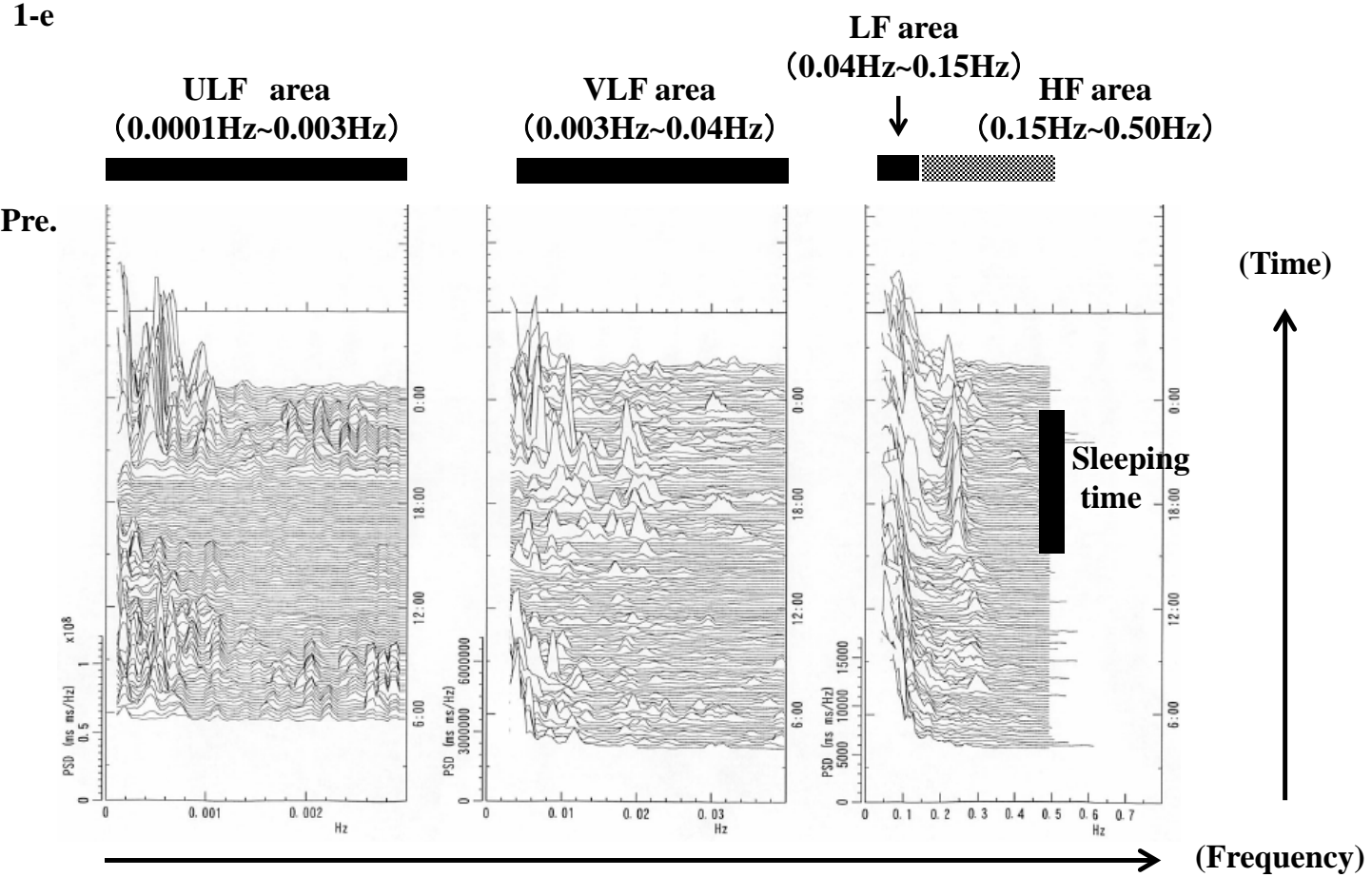

1-f

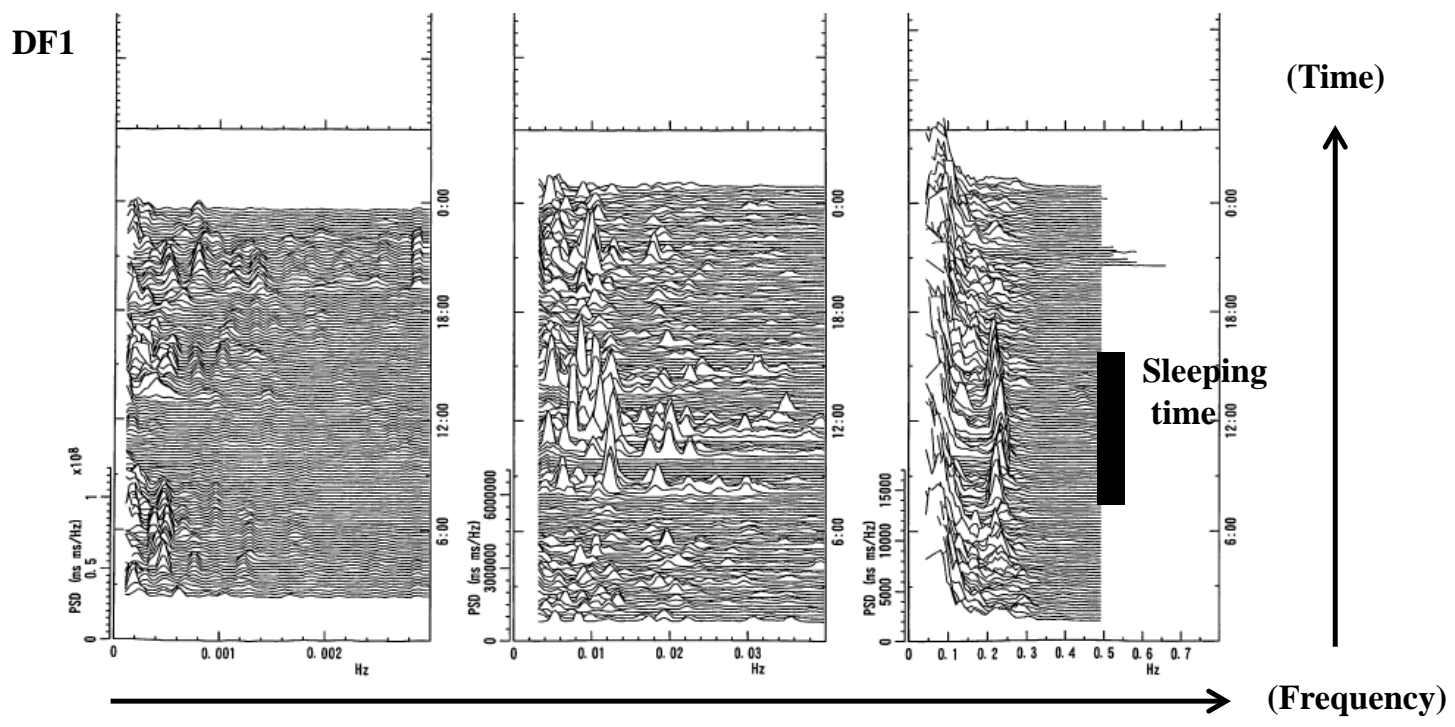


1-g

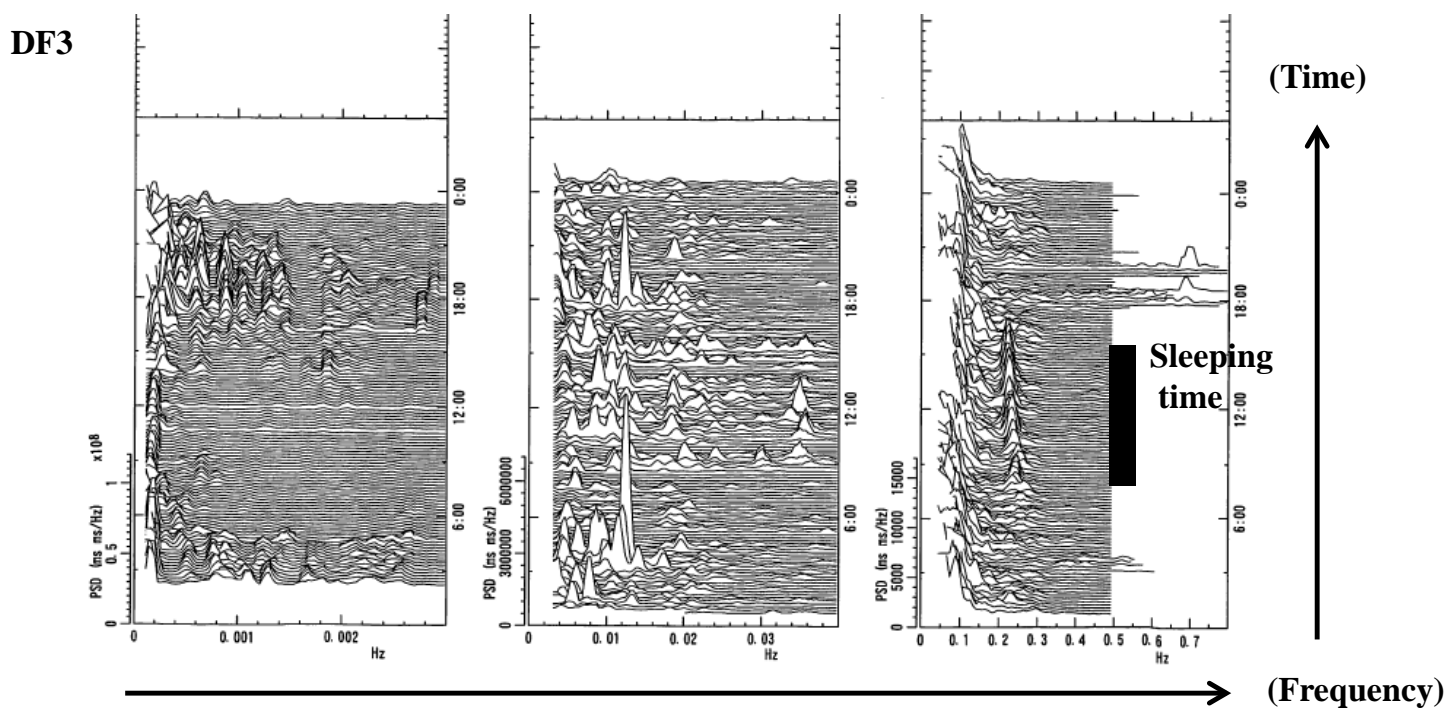

1-h

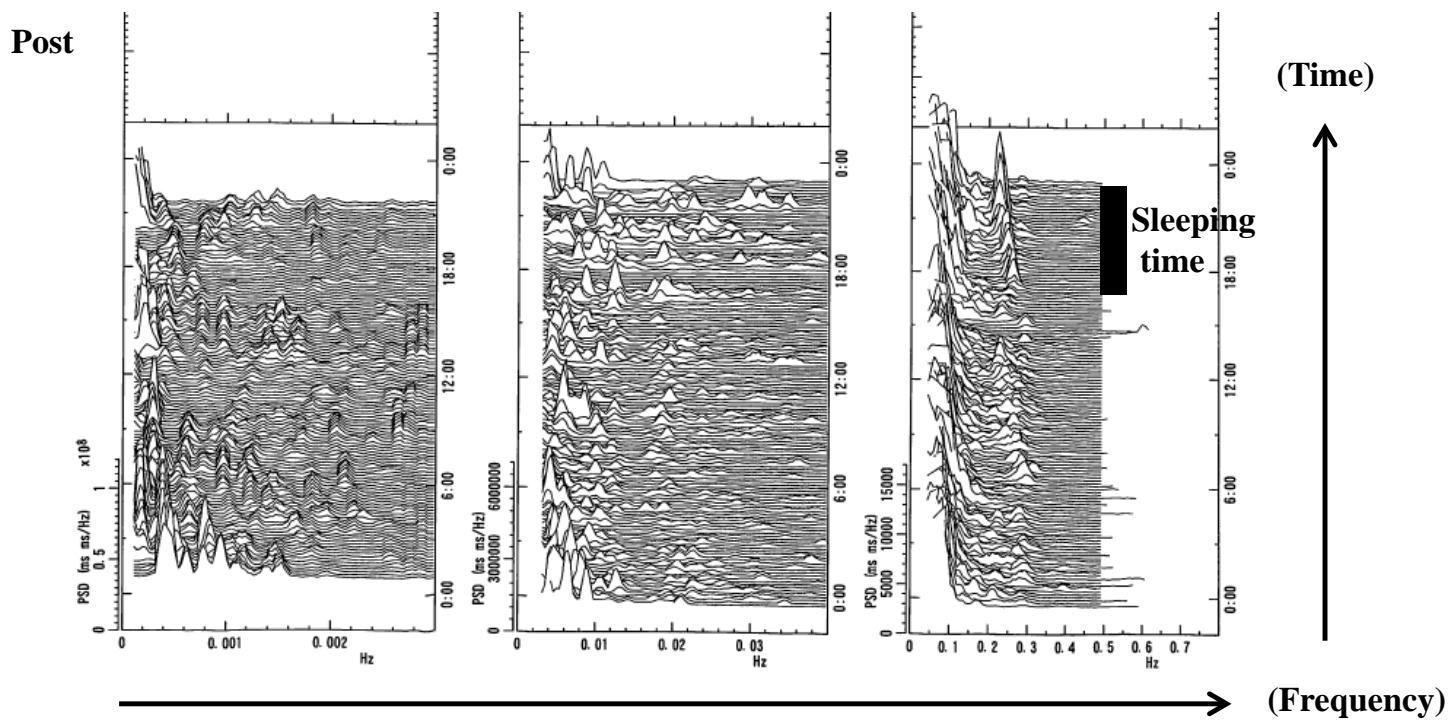


1-i

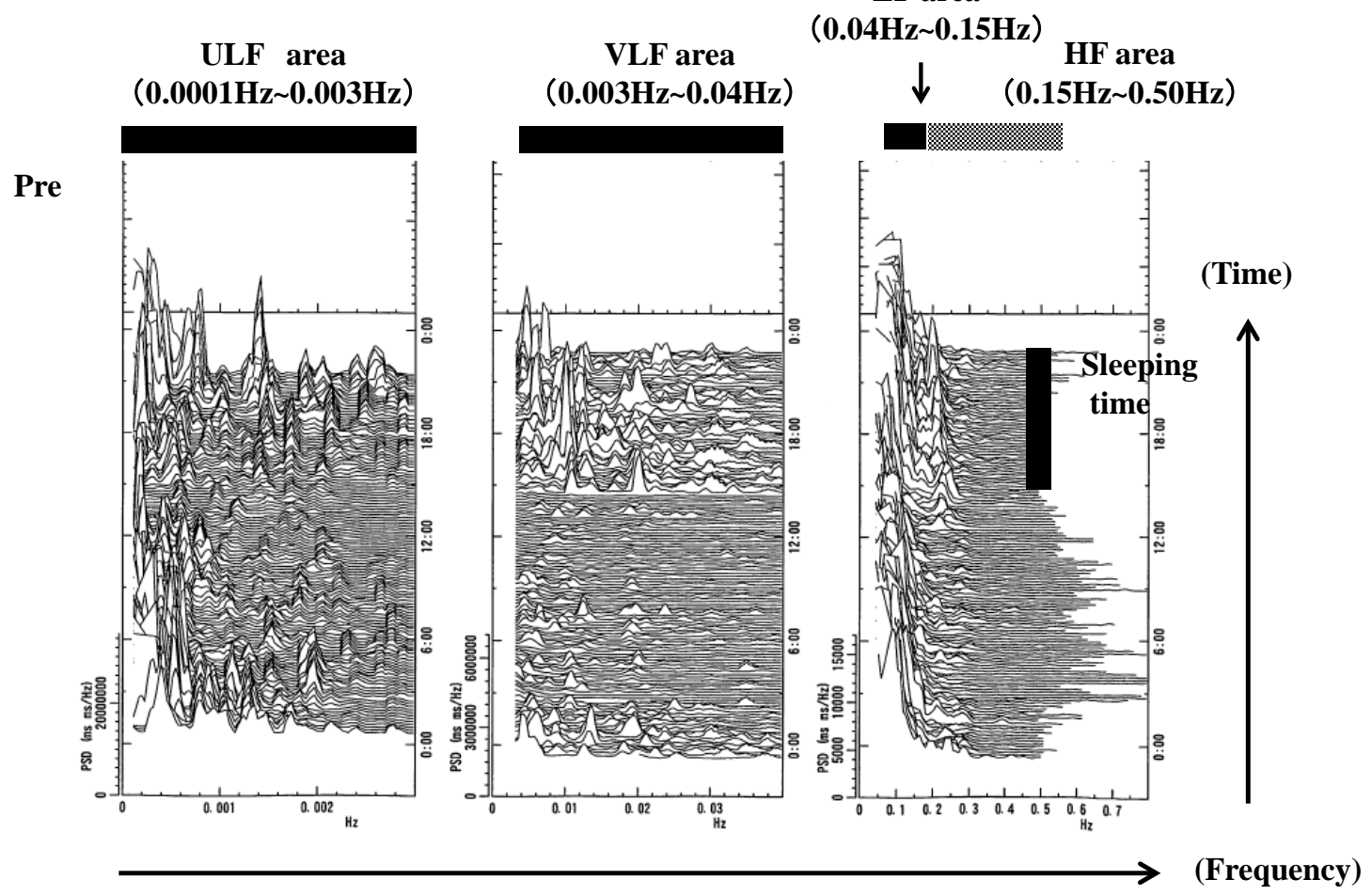

1-j
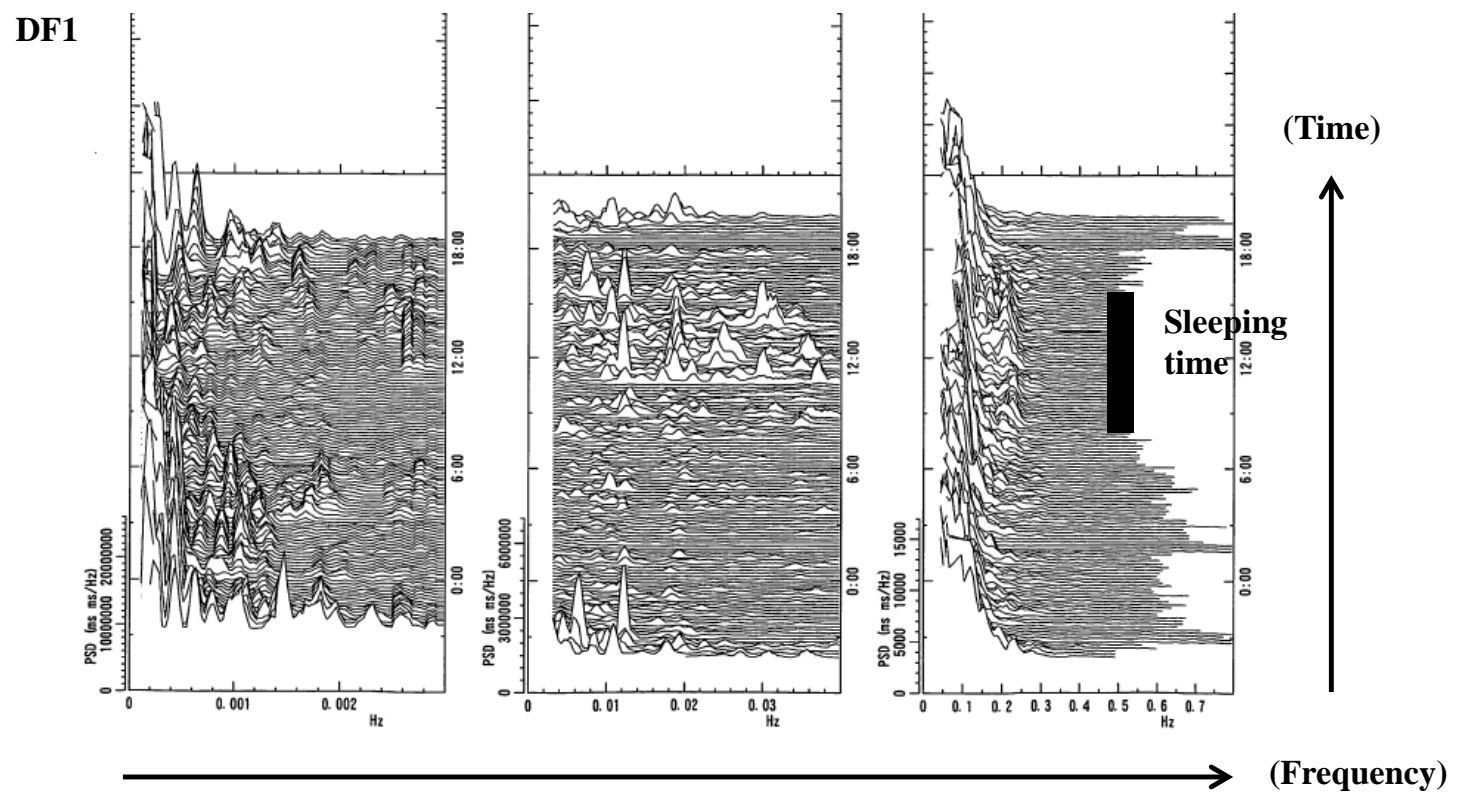
1-k
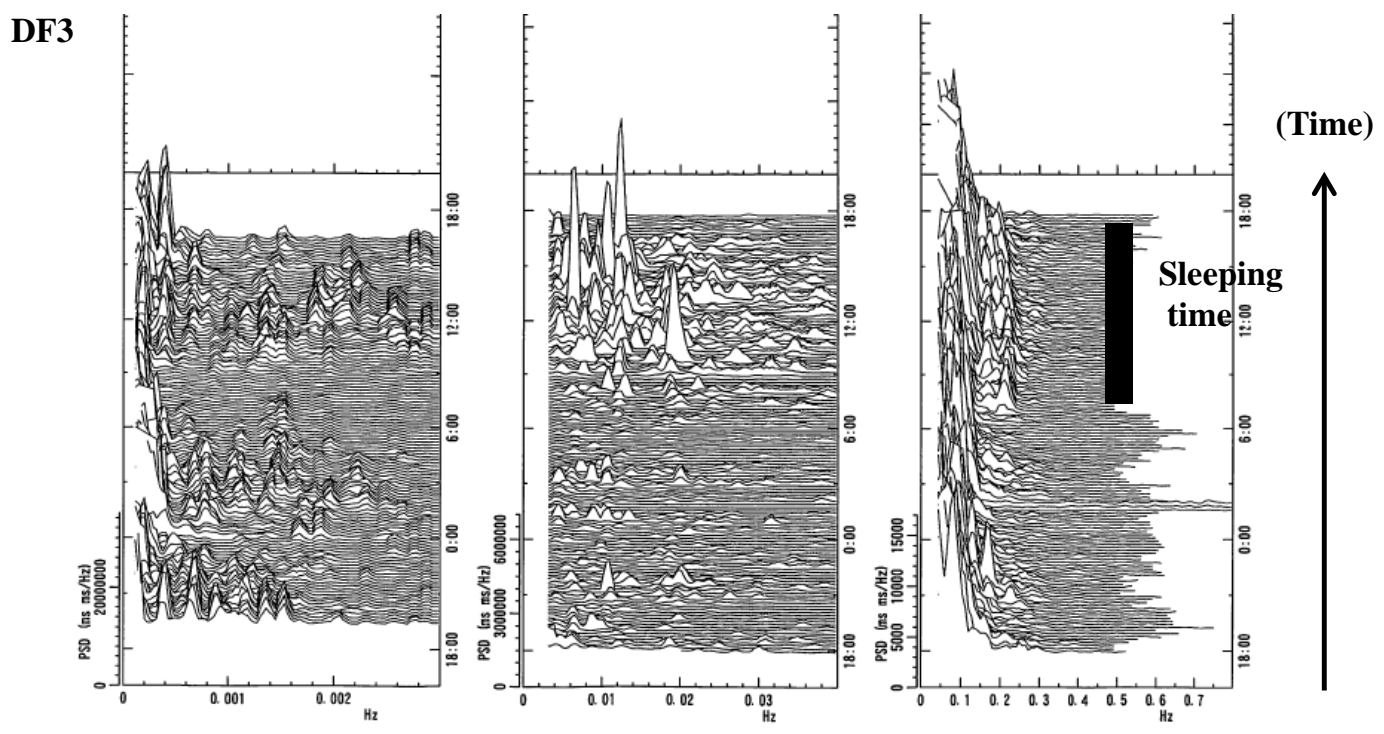

(Frequency)

1-I
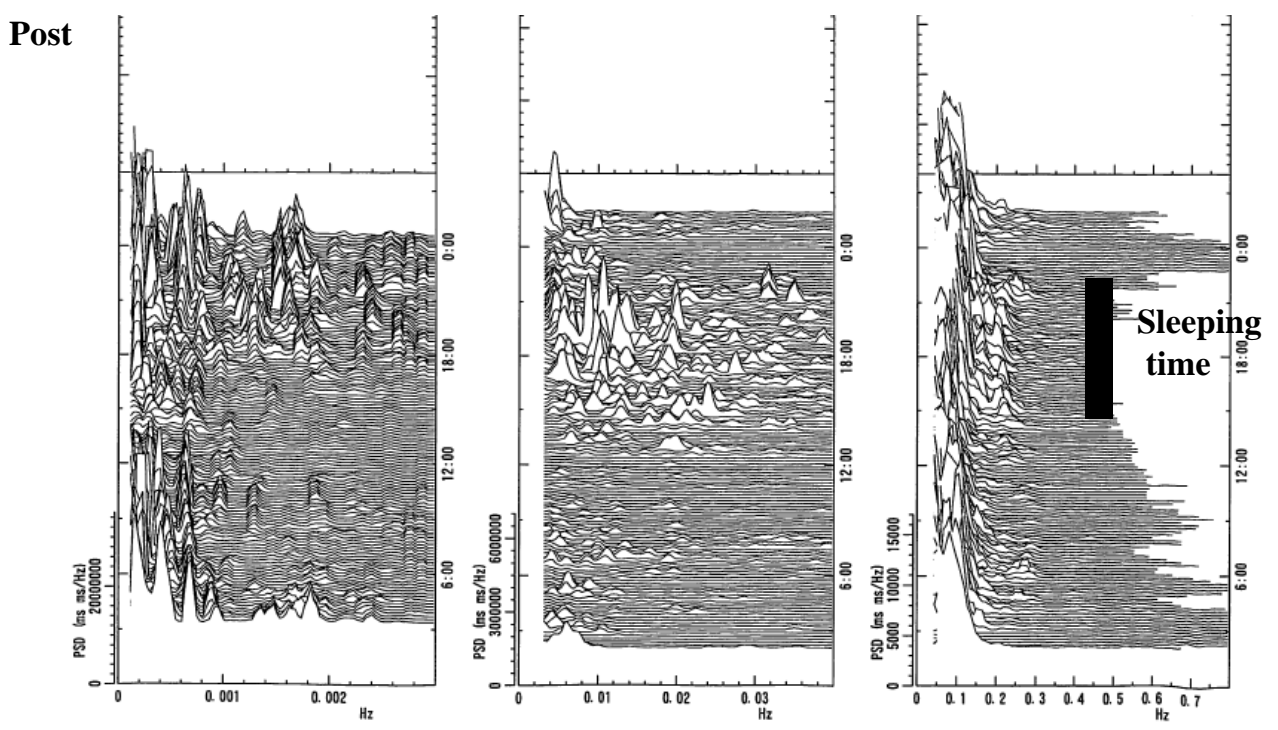

(Time)

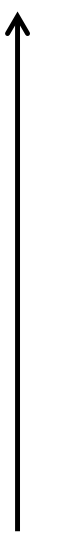

(Frequency)

Figure legends

Fig. 1. Change of autonomic nerve function in space in 3 subjects

Results of spectral analyses of HRV are shown in Figs.1a-l. The 24-hr spectral arrays analyzed using MemCalc/Chiram are shown in three dimensions. Results are shown for 3 different subjects 
in Figs. 1a-d, 1e-h and 1i-l, respectively. The figures portray the spectral charts before launch (Pre), in the early flight (DF1), in the late flight (DF3) and after return (Post), respectively. The left, middle and right graphs portray the spectral arrays in the ULF $(0.0001-0.003 \mathrm{~Hz})$, VLF (0.003$0.04 \mathrm{~Hz})$, and LF (0.15-0.04 Hz) and HF (0.04-0.50 Hz) domains, respectively. We sequentially analyzed Holter electrocardiographic records with the Maximum Entropy Method. Intervals of 300 s (5 min) were used for assessing the VLH, LF, and HF spectral power densities, and intervals of $10,800 \mathrm{~s}$ (180 $\mathrm{min})$ in the case of the ULF spectral power density. All intervals were progressively displaced by 5 min throughout the record. In view of the longer interval used for ULF, the start time is 90 min later than that for VLF, LF, and HF along the time axis. A right black bar indicates the sleeping time.

Figs. 1a-1d show results for one subject (ID01) who developed strong bradycardia at DF1 (NN intervals increased by more than 10\%). Figs. 1e-1h present results from another subject (ID04), who developed mild bradycardia at DF1. Figs. 1i-1l portray results of yet another subject (ID05), who developed mild tachycardia at DF1. 
Pre.
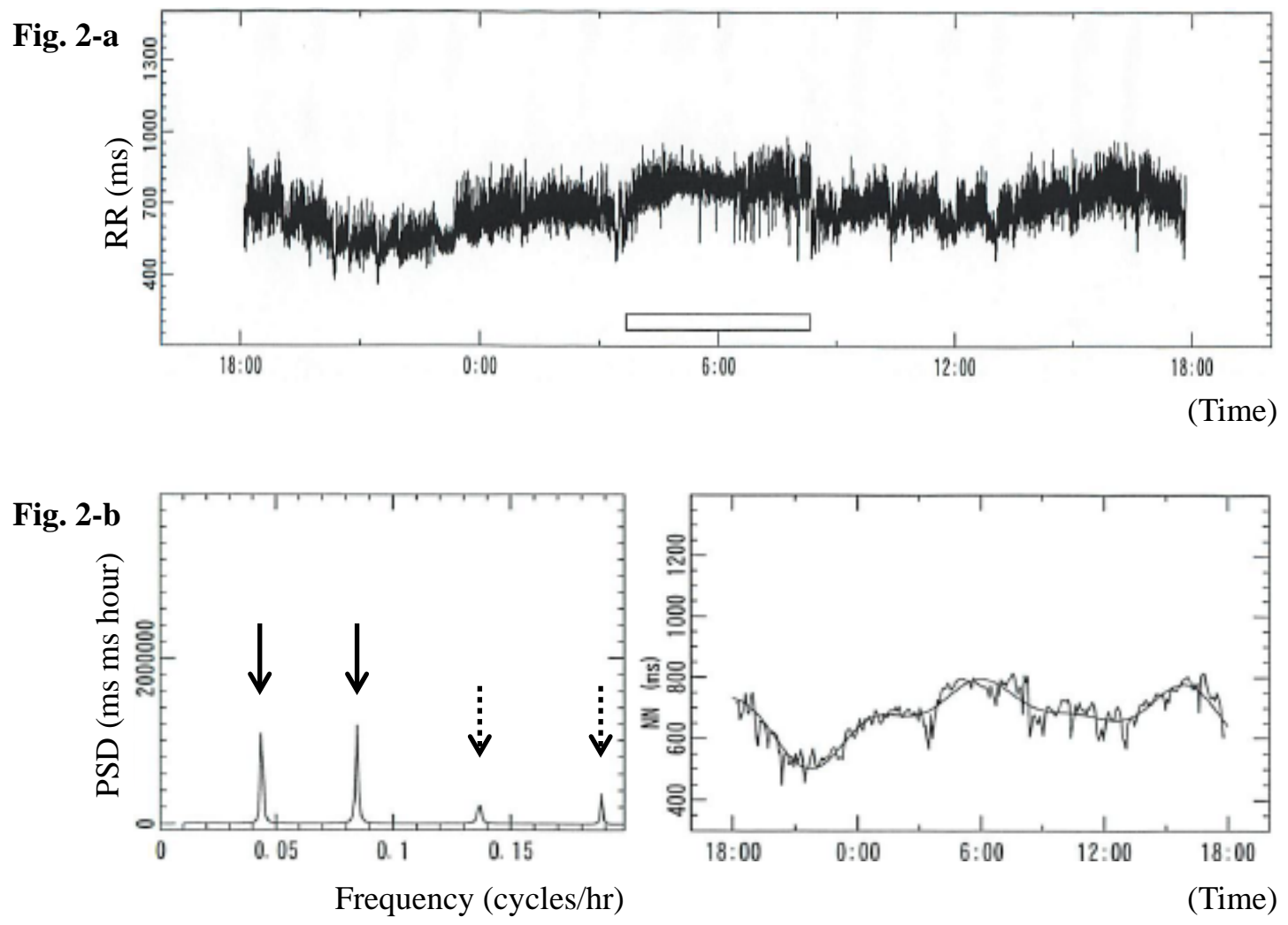

DF1.

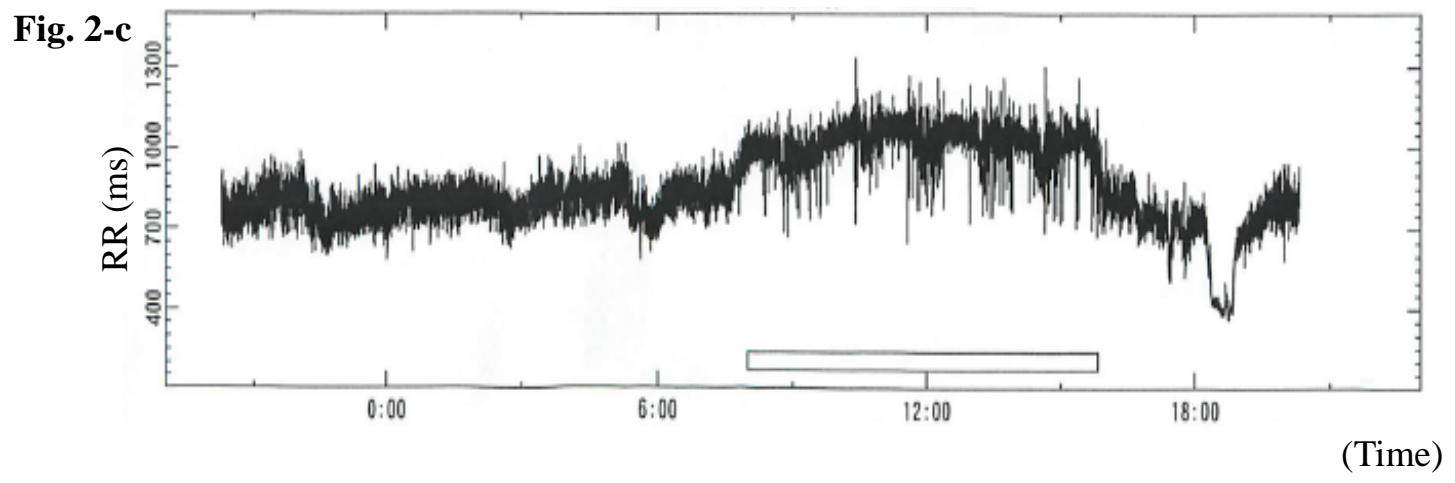

Fig. 2-d
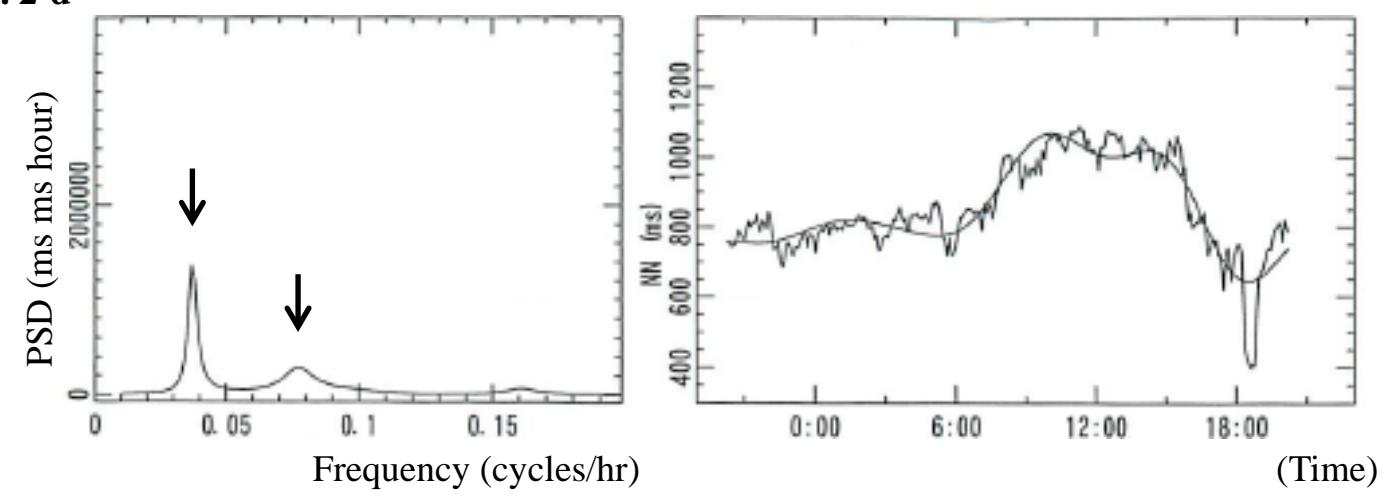
DF3.

Fig. 2-e

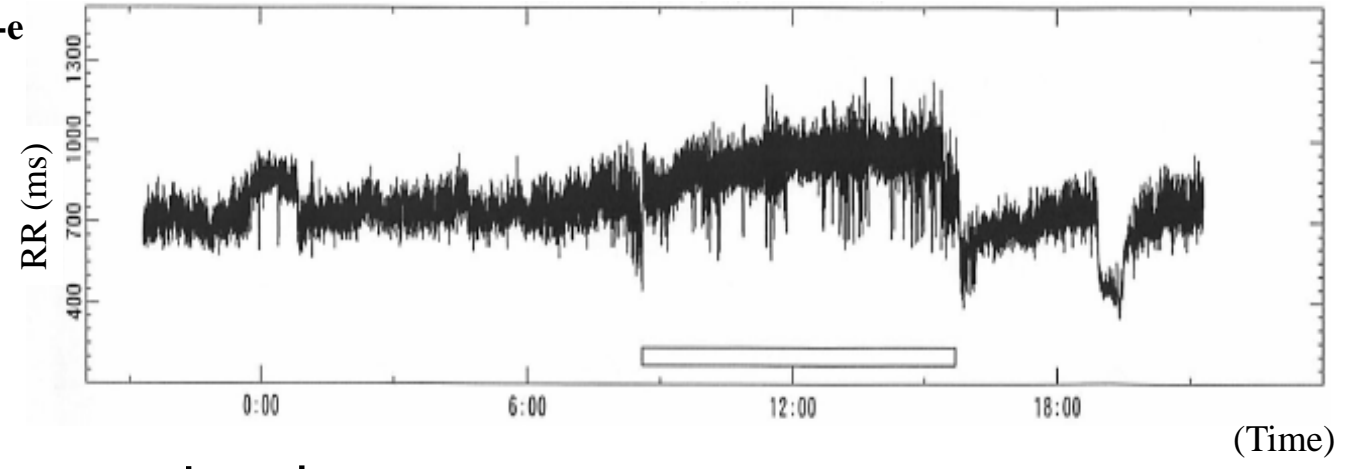

Fig. 2-f
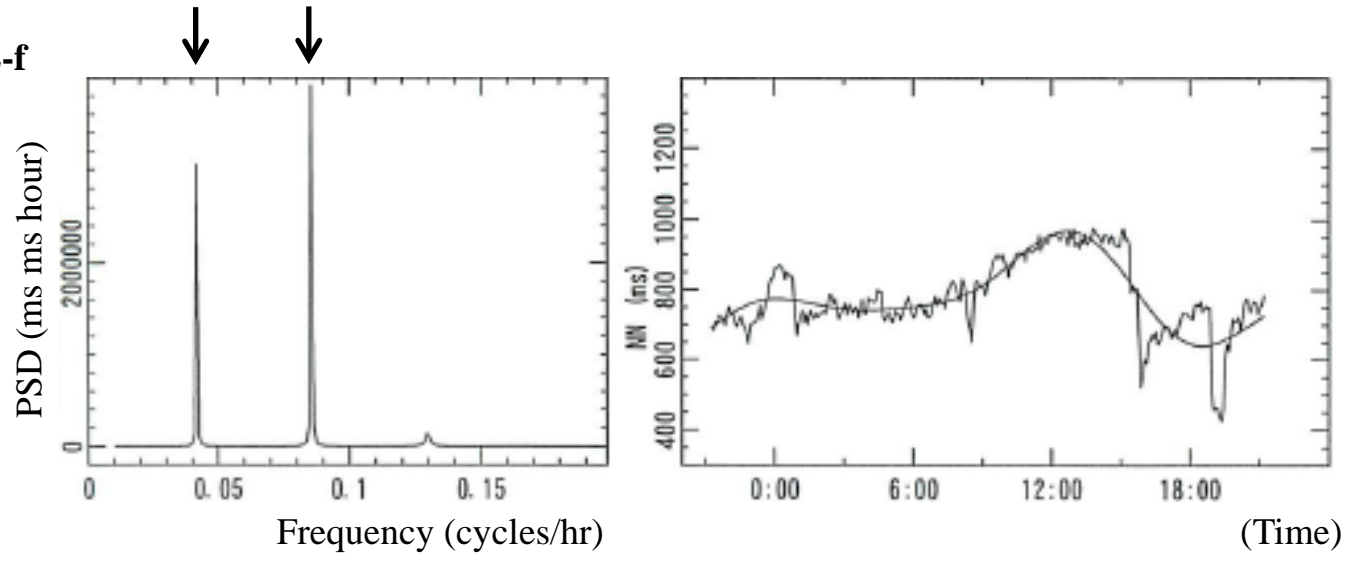

Post.

Fig. 2-g

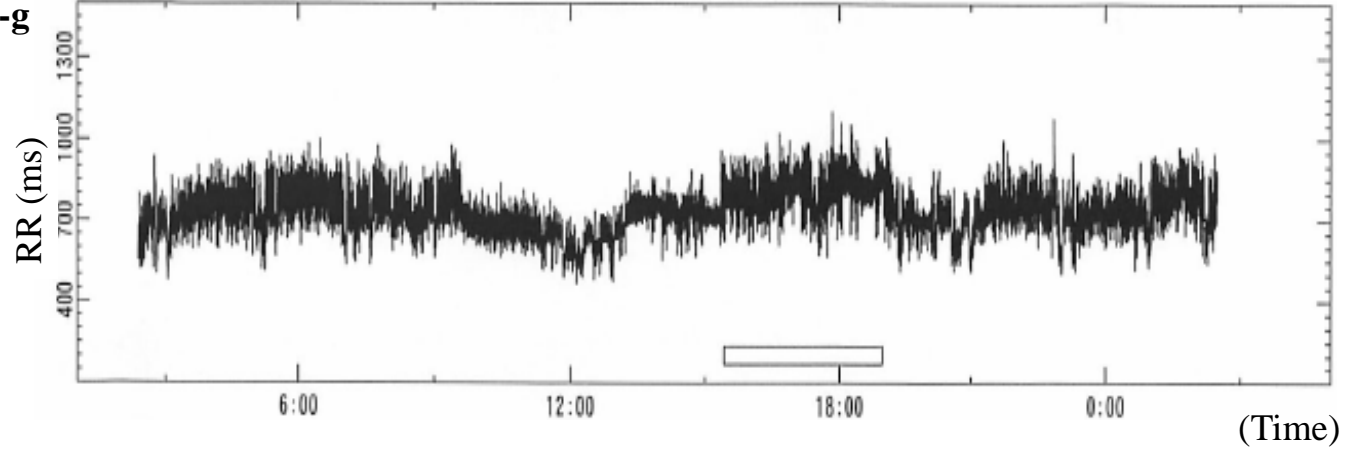

Fig. 2-h
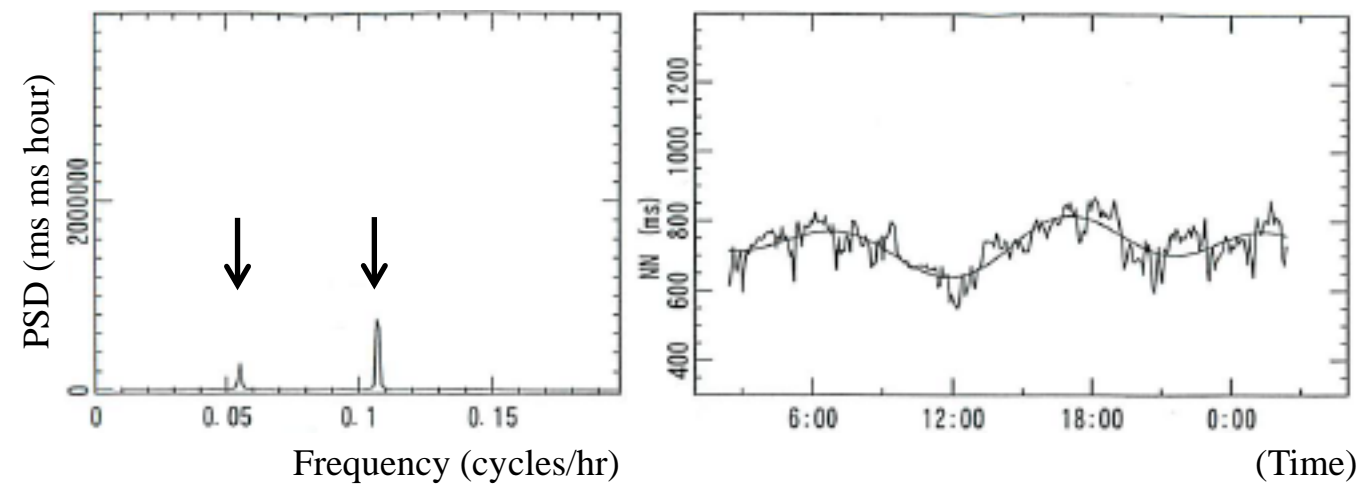
Fig. 2a. Sample of Preflight RR-interval data for 24 hr without noise or artifacts (NN intervals)

Time is shown on the horizontal axis and NN intervals on the vertical axis. A white bar indicates the sleeping time. In this case, after falling asleep, NN intervals gradually extended to 800-900 ms, and shortened to 700 ms after getting up.

Fig. 2b. Frequency-domain analysis of $\mathrm{NN}$ intervals (left) and circadian waveform (right) before flight.

In the left panel, the power-spectral density (PSD hr/min/min) is plotted as a function of frequency (cycles/hr). Detected components have periods of $22.71 \mathrm{hr}$ and $11.80 \mathrm{hr}$ (about half of $22.71 \mathrm{hr}$ ), accounting for $31.6 \%$ and $34.9 \%$ of the total power (variance), respectively. They represent the circadian rhythm characterizing NN intervals. In addition, 7.31-hr and 5.30-hr components were also detected. The signal reconstructed (at 5-min intervals) based on these four components (accounting for $80.5 \%$ of the total power) fitted to the NN intervals by least-squares is shown with the original data (right panel), illustrating the circadian variation in the data.

\section{Fig. 2c. NN intervals for 24 hrs during DF1}

At one month after launch (DF1), NN intervals varied around 700 ms when awake, similar to preflight, but lengthened to 900-1100 ms during sleep, which is longer than during pre-flight. A transient drop in $\mathrm{NN}$ intervals is observed from 18:30 to 19:00 during exercise, recovering thereafter.

Fig. 2d. Frequency-domain analysis of $\mathrm{NN}$ intervals (left) and circadian waveform (right) during DF1

The MEM analysis (left) detects two peaks corresponding to periods of $26.79 \mathrm{hr}$ (51.9\% power) and $12.93 \mathrm{hr}$ (23.3\% power), approximately half a day. The circadian waveform thus reconstructed 
(at 5-min intervals) by the least-squares fit of cosine curves with these periods to the data are shown with the original data (right).

Fig. 2e. NN intervals for 24 hr during DF3.

During DF3, NN intervals average about 700 ms while awake, prolonged to 850-1000 ms during sleep, and shortened to 500-600 ms at the time of awakening.

Fig. 2f. Frequency-domain analysis of NN intervals (left) and circadian waveform (right) during DF3.

The MEM analysis (left) detects two major peaks corresponding to periods of $24.00 \mathrm{hr}$ (35.6\% power) and $11.68 \mathrm{hr}$ (30.2\% power) (left), very close to 24 and $12 \mathrm{hr}$, suggesting that the biological clock mechanism that modulates the circadian rhythm recovered from the disruption associated with the flight. The reconstructed signal (right) shows a clear circadian variation.

Fig. 2g. Post NN intervals for 24 hr after return to Earth

In the Post term, NN intervals averaged 800-950 ms during sleep, similar to pre-flight and much less prolonged than in space. In this case, the sleep span was very short (about $3.5 \mathrm{hr}$ ).

Fig. 2h. Frequency-domain analysis of $\mathrm{NN}$ intervals (left) and circadian waveform (right) post-flight

The MEM analysis (left) detects two peaks with periods of $18.17 \mathrm{hr}(16.4 \%$ power) and $9.34 \mathrm{hr}$ (39.8\% power), and barely any other periodic components. Although these peaks were clearly detected, their spectral density was low. Overall, the spectral power was markedly decreased. The 9.34-hr component was the most prominent. A clear circadian pattern could not be observed from the reconstructed signal (right). 
Fig.3

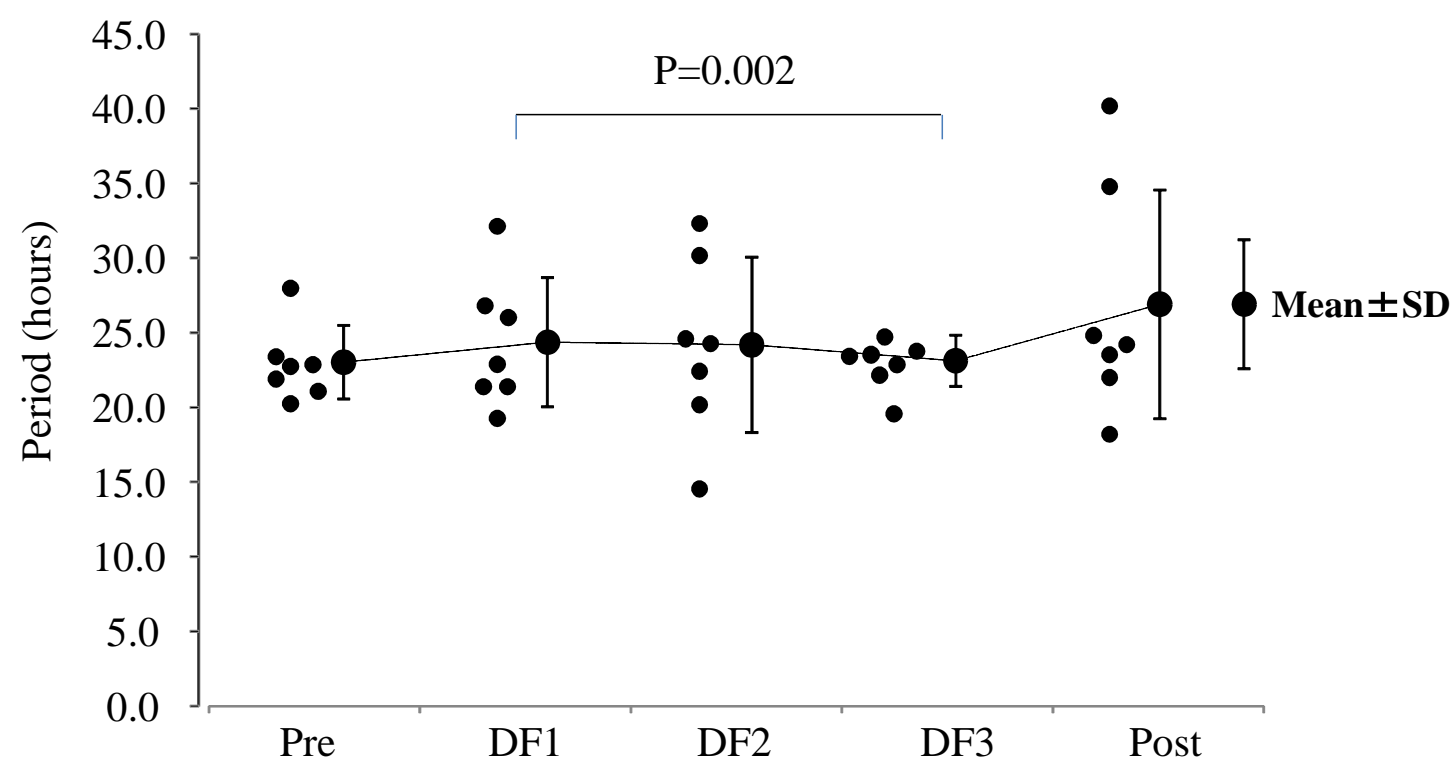

Fig. 3. Comparison of astronauts' circadian period estimates before, during and after flight MEM estimates of the circadian period of NN intervals of all seven astronauts are shown (small dots) together with their mean (large dots) and SD (vertical bars). The average period was within the defined circadian range of 24 $\pm 4 \mathrm{hr}$ before, during, and after flight (Pre: 22.36; DF1: 25.46; DF2: 23.17; DF3: 22.46; Post: 26.16; NS). The standard deviation (SD) of the circadian period was smaller during DF3 (Pre: 2.50; DF1: 4.37; DF2: 5.97; DF3: 1.75; Post: 7.18 hr, P=0.002, Wilcoxon paired test from DF3 vs. DF1 comparison). 
Table 1. Change in indexes of heart rate variability of all astronauts

\begin{tabular}{|c|c|c|c|c|c|c|c|c|c|}
\hline \multicolumn{10}{|l|}{ Pre-Flight } \\
\hline & ID-01 & ID-02 & ID-03 & ID-04 & ID-05 & ID-06 & ID-07 & All-value mean & All-value SD \\
\hline NN-interval (msec) & 682.72 & 788.38 & 775.06 & 1181.65 & 970.47 & 934.22 & 815.25 & 878.25 & 165.64 \\
\hline CVRR (\%) & 6.63 & 6.66 & 7.17 & 7.83 & 3.64 & 8.86 & 6.63 & 6.78 & 1.61 \\
\hline rMSSD (msec) & 23.18 & 16.76 & 23.12 & 34.83 & 16.28 & 33.77 & 23.14 & 24.44 & 7.37 \\
\hline SDNN (msec) & 96.20 & 113.80 & 128.90 & 212.60 & 85.40 & 211.10 & 72.30 & 131.47 & 57.89 \\
\hline SDANN (5-min) (msec) & 81.50 & 102.40 & 105.70 & 185.60 & 76.30 & 196.20 & 102.90 & 121.51 & 48.81 \\
\hline TI (HRVI) & 31.02 & 26.90 & 30.33 & 57.92 & 25.30 & 47.75 & 29.63 & 35.55 & 12.33 \\
\hline TF-component (msec $\left.{ }^{2}\right)$ & 2798.62 & 5478.45 & 5668.79 & 17935.20 & 3580.94 & 12223.90 & 5667.10 & 7621.86 & 5467.57 \\
\hline ULF-component (msec ${ }^{2}$ ) & 1218.82 & 3299.31 & 3148.91 & 11271.10 & 2647.89 & 6219.51 & 3221.12 & 4432.38 & 3363.32 \\
\hline VLF-component (msec ${ }^{2}$ ) & 972.25 & 1381.63 & 1676.81 & 5677.11 & 801.14 & 4718.29 & 1636.15 & 2409.05 & 1951.57 \\
\hline LF-component $\left(\mathrm{msec}^{2}\right)$ & 570.11 & 678.38 & 760.99 & $890.439 \square$ & 164.57 & 1380.75 & 602.27 & 692.84 & 395.07 \\
\hline HF-component (msec ${ }^{2}$ ) & 108.32 & 55.24 & 164.46 & 159.06 & 37.01 & 202.87 & 128.26 & 122.17 & 60.03 \\
\hline LF/HF ratio $\left(\mathrm{msec}^{2}\right)$ & 5.67 & 13.53 & 4.63 & 6.77 & 4.92 & 7.81 & 6.06 & 7.06 & 3.05 \\
\hline Max power cycle(hour) & 22.71 & 23.37 & 22.82 & 27.95 & 20.22 & 21.87 & 21.04 & 22.85 & 2.50 \\
\hline Difference between 24hr(hour) & 1.29 & 0.63 & 1.18 & 3.95 & 3.78 & 2.13 & 2.96 & 2.27 & 1.32 \\
\hline \multicolumn{10}{|l|}{ DF1(Stay ISS within 1 month) } \\
\hline & ID-01 & ID-02 & ID-03 & ID-04 & ID-05 & ID-06 & ID-07 & All-value mean & All-value SD \\
\hline NN-interval (msec) & 860.11 & 878.81 & 914.69 & 1242.62 & 1018.58 & 891.89 & 758.35 & 937.87 & 154.81 \\
\hline CVRR (\%) & 5.30 & 5.56 & 7.33 & 6.23 & 3.36 & 7.64 & 6.16 & 5.94 & 1.42 \\
\hline rMSSD (msec) & 20.36 & 17.84 & 29.72 & 33.57 & 11.88 & 27.97 & 22.70 & 23.44 & 7.50 \\
\hline SDNN (msec) & 152.90 & 152.10 & 136.60 & 181.10 & 114.10 & 208.90 & 135.50 & 154.46 & 31.59 \\
\hline SDANN (5-min) (msec) & 15.62 & 123.60 & 114.30 & 158.80 & 104.50 & 187.30 & 111.50 & 116.52 & 53.55 \\
\hline TI (HRVI) & 27.35 & 32.53 & 41.43 & 54.52 & 28.74 & 48.68 & 33.92 & 38.17 & 10.36 \\
\hline TF-component $\left(\mathrm{msec}^{2}\right)$ & 3867.57 & 6557.30 & 6905.13 & 9713.10 & 2285.06 & 9102.30 & 4350.63 & 6111.58 & 2754.47 \\
\hline ULF-component (msec ${ }^{2}$ ) & 1832.88 & 4612.91 & 3331.77 & 4482.10 & 1239.25 & 4890.23 & 2591.10 & 3282.89 & 1446.29 \\
\hline VLF-component (msec $\left.{ }^{2}\right)$ & 1476.01 & 1372.66 & 2440.43 & 4136.25 & 875.69 & 2974.86 & 1163.82 & 2062.82 & 1176.98 \\
\hline LF-component $\left(\mathrm{msec}^{2}\right)$ & 390.99 & 535.92 & 1202.70 & 803.88 & 144.56 & 1156.72 & 555.01 & 684.25 & 392.07 \\
\hline HF-component (msec $\left.{ }^{2}\right)$ & 87.02 & 56.66 & 237.44 & 160.46 & 22.07 & 150.69 & 92.62 & 115.28 & 72.63 \\
\hline LF/HF ratio $\left(\mathrm{msec}^{2}\right)$ & 5.77 & 10.55 & 5.07 & 5.74 & 7.65 & 7.48 & 6.60 & 6.98 & 1.84 \\
\hline Max power cycle (hour) & 26.77 & 21.34 & 22.85 & 25.98 & 21.35 & 19.25 & 32.10 & 24.23 & 4.37 \\
\hline Difference between 24hr(hour) & 2.77 & 2.66 & 1.15 & 1.98 & 2.65 & 4.75 & 8.10 & 3.44 & 2.33 \\
\hline
\end{tabular}




\begin{tabular}{|c|c|c|c|c|c|c|c|c|c|}
\hline \multicolumn{10}{|l|}{ DF2(Stay ISS within 2 month) } \\
\hline & ID-01 & ID-02 & ID-03 & ID-04 & ID-05 & ID-06 & ID-07 & All-value mean & All-value SD \\
\hline NN-interval (msec) & 860.05 & 932.67 & 780.94 & 1128.38 & 1083.32 & 952.93 & 802.76 & 934.44 & 133.27 \\
\hline CVRR (\%) & 5.23 & 5.09 & 6.60 & 6.17 & 3.31 & 7.50 & 6.25 & 5.74 & 1.35 \\
\hline rMSSD (msec) & 18.38 & 19.72 & 23.48 & 27.01 & 14.99 & 33.19 & 23.32 & 22.87 & 6.00 \\
\hline SDNN (msec) & 108.70 & 44.70 & 101.10 & 255.00 & 152.60 & 152.60 & 93.00 & 129.67 & 66.56 \\
\hline $\operatorname{SDANN}(5-\mathrm{min})(\mathrm{msec})$ & 99.00 & 89.30 & 78.90 & 212.20 & 133.10 & 133.10 & 77.60 & 117.60 & 47.74 \\
\hline TI (HRVI) & 24.61 & 26.52 & 21.92 & 48.53 & 26.90 & 26.90 & 21.73 & 28.16 & 9.25 \\
\hline TF-component (msec $\left.{ }^{2}\right)$ & 3055.00 & 4033.33 & 5327.73 & 11712.20 & 3277.24 & 8750.60 & 3318.10 & 5639.17 & 3338.72 \\
\hline ULF-component ( $\mathrm{msec}^{2}$ ) & 973.28 & 2161.60 & 2954.44 & 8314.70 & 2262.37 & 3792.84 & 1185.15 & 3092.05 & 2498.86 \\
\hline VLF-component ( $\mathrm{msec}^{2}$ ) & 1545.98 & 1338.39 & 1337.30 & 2834.23 & 823.68 & 3421.49 & 1451.65 & 1821.82 & 936.46 \\
\hline LF-component (msec $\left.{ }^{2}\right)$ & 374.15 & 491.46 & 849.36 & 624.16 & 208.17 & 1262.92 & 664.26 & 639.21 & 344.46 \\
\hline HF-component $\left(\mathrm{msec}^{2}\right)$ & 72.26 & 74.17 & 161.35 & 105.25 & 30.93 & 182.79 & 100.67 & 103.92 & 52.83 \\
\hline LF/HF ratio $\left(\mathrm{msec}^{2}\right)$ & 6.12 & 7.37 & 5.26 & 6.20 & 7.69 & 7.22 & 7.08 & 6.71 & 0.86 \\
\hline Max power cycle (hour) & 22.39 & 14.52 & 24.57 & 20.14 & 32.28 & 30.14 & 24.25 & 24.04 & 5.97 \\
\hline Difference between $24 \mathrm{hr}$ (hour) & 1.61 & 9.48 & 0.57 & 3.86 & 8.28 & 6.14 & 0.25 & 4.31 & 3.74 \\
\hline \multicolumn{10}{|l|}{ DF3 (Stay ISS about 6 month) } \\
\hline & ID-01 & ID-02 & ID-03 & ID-04 & ID-05 & ID-06 & ID-07 & All-value mean & All-value SD \\
\hline NN-interval (msec) & 782.04 & 926.68 & 808.02 & 1181.54 & 1060.77 & 941.45 & 858.48 & 937.00 & 142.63 \\
\hline CVRR (\%) & 6.02 & 5.39 & 6.98 & 7.00 & 3.40 & 7.81 & 6.63 & 6.17 & 1.45 \\
\hline rMSSD (msec) & 33.20 & 20.07 & 25.85 & 32.53 & 15.13 & 33.07 & 27.14 & 26.71 & 7.02 \\
\hline SDNN (msec) & 122.00 & 160.80 & 96.80 & 246.10 & 121.40 & 175.70 & 137.40 & 151.46 & 49.31 \\
\hline $\operatorname{SDANN}(5-\mathrm{min})(\mathrm{msec})$ & 107.90 & 124.00 & 79.80 & 218.10 & 113.60 & 155.80 & 108.30 & 129.64 & 45.11 \\
\hline TI (HRVI) & 21.91 & 29.31 & 21.30 & 44.02 & 32.12 & 32.45 & 32.33 & 30.49 & 7.66 \\
\hline TF-component (msec ${ }^{2}$ ) & 4448.29 & 6107.63 & 4485.87 & 13555.60 & 2896.30 & 10408.90 & 5735.69 & 6805.47 & 3794.71 \\
\hline ULF-component ( $\mathrm{msec}^{2}$ ) & 2339.61 & 4015.25 & 1792.26 & 7803.20 & 1755.23 & 4210.80 & 2989.02 & 3557.91 & 2114.75 \\
\hline VLF-component ( $\mathrm{msec}^{2}$ ) & 1474.85 & 1428.86 & 1598.59 & 4363.77 & 937.50 & 4366.08 & 1724.17 & 2270.55 & 1451.52 \\
\hline LF-component (msec ${ }^{2}$ ) & 353.10 & 588.24 & 977.74 & 750.28 & 199.90 & 1231.13 & 843.58 & 706.28 & 357.08 \\
\hline HF-component $\left(\mathrm{msec}^{2}\right)$ & 183.09 & 68.65 & 235.93 & 124.83 & 31.04 & 206.38 & 127.50 & 139.63 & 73.95 \\
\hline LF/HF ratio $\left(\mathrm{msec}^{2}\right)$ & 3.33 & 9.29 & 4.14 & 6.32 & 6.99 & 6.34 & 7.29 & 6.24 & 1.99 \\
\hline Max power cycle (hour) & 23.98 & 22.14 & 23.91 & 23.74 & 24.61 & 22.83 & 19.45 & 22.95 & 1.75 \\
\hline Difference between 24hr(hour) & 0.02 & 1.86 & 0.09 & 0.26 & 0.61 & 1.17 & 4.55 & 1.22 & 1.61 \\
\hline \multicolumn{10}{|c|}{ Post(Return to the Earth within about 3 month) } \\
\hline & ID-01 & ID-02 & ID-03 & ID-04 & ID-05 & ID-06 & ID-07 & All-value mean & All-value SD \\
\hline NN-interval (msec) & 732.79 & 902.86 & 778.10 & 1147.67 & 965.97 & 840.19 & 842.71 & 887.18 & 137.98 \\
\hline CVRR (\%) & 6.70 & 6.33 & 7.28 & 7.00 & 3.65 & 8.52 & 7.31 & 6.69 & 1.50 \\
\hline rMSSD (msec) & 18.95 & 22.98 & 23.84 & 31.68 & 12.72 & 27.34 & 28.88 & 23.77 & 6.42 \\
\hline SDNN (msec) & 82.70 & 101.90 & 112.10 & 176.00 & 166.70 & 220.30 & 162.50 & 146.03 & 48.69 \\
\hline $\operatorname{SDANN}(5-\mathrm{min})$ (msec) & 63.40 & 82.00 & 94.40 & 152.10 & 148.40 & 204.90 & 124.10 & 124.19 & 48.72 \\
\hline TI (HRVI) & 24.86 & 30.44 & 35.15 & 35.83 & 31.17 & 51.24 & 35.01 & 34.81 & 8.19 \\
\hline TF-component $\left(\mathrm{msec}^{2}\right.$ ) & 3532.24 & 6024.41 & 5809.56 & 12490.20 & 4228.36 & 9527.30 & 7091.27 & 6957.62 & 3125.21 \\
\hline ULF-component ( $\mathrm{msec}^{2}$ ) & 1563.18 & 3521.32 & 3118.71 & 7731.10 & 3335.56 & 4531.02 & 3826.49 & 3946.77 & 1897.31 \\
\hline VLF-component $\left(\mathrm{msec}^{2}\right.$ ) & 1395.54 & 1735.05 & 1720.79 & 3871.84 & 725.17 & 3600.71 & 2227.05 & 2182.31 & 1156.41 \\
\hline LF-component $\left(\mathrm{msec}^{2}\right)$ & 508.64 & 687.43 & 783.85 & 817.17 & 155.27 & 1070.61 & 859.51 & 697.50 & 293.55 \\
\hline HF-component $\left(\mathrm{msec}^{2}\right)$ & 94.39 & 102.08 & 164.09 & 139.99 & 23.48 & 121.71 & 193.69 & 119.92 & 54.88 \\
\hline LF/HF ratio $\left(\mathrm{msec}^{2}\right.$ ) & 6.22 & 8.11 & 4.78 & 6.85 & 7.65 & 8.88 & 4.86 & 6.76 & 1.58 \\
\hline Max power cycle (hour) & 18.17 & 24.17 & 40.16 & 34.76 & 21.98 & 23.51 & 24.81 & 26.79 & 7.76 \\
\hline Difference between $24 \mathrm{hr}$ (hour) & 5.83 & 0.17 & 16.16 & 10.76 & 2.02 & 0.49 & 0.81 & 5.18 & 6.17 \\
\hline
\end{tabular}

CVRR: Coefficent of Variationof R-R intervvals

rMSSD: Root mean square differences of successive R-R (heartbeat) intervals

SDNN: Standard Deviation of The NN(RR) Intervals

SDANN (5min) : Standard Deviation of the Averages of NN (Normal Sinus to Normal Sinus) Intervals in All 5-Minute Segments of a 24-Hour Recording.

TI: Triangular index

TF: Total frequency

ULF: Ultra low frequency

VLF: Very low frequency

LF: Low frequency

HF: High frequency

L/H: Low frequency/high frequency ratio

Table 1. Change in indexes of heart rate variability of all astronauts

The change in each index of heart rate variability of the seven astronauts is presented in Table 1. At DF1, after one month in space, five of the seven subjects increased NN intervals (ID01-ID05), and two decreased NN intervals (ID06-ID07), as compared to conditions before flight. The difference in HRV response patterns suggests that the capability of adapting to the space environment varies among different individuals. 